\title{
Danofloxacin Treatment Alters the Diversity and Resistome Profile of Gut Microbiota in Calves
}

\author{
Ashenafi Feyisa Beyi ${ }^{1}{ }^{\mathbb{D}}$, Debora Brito-Goulart ${ }^{1}$, Tyler Hawbecker ${ }^{2}$, Clare Slagel $^{2}$, Brandon Ruddell ${ }^{1}$, \\ Alan Hassall ${ }^{3}$, Renee Dewell ${ }^{4}\left(\mathbb{D}\right.$, Grant Dewell ${ }^{3}$ (D) , Orhan Sahin ${ }^{3}$, Qijing Zhang ${ }^{1}$ and Paul J. Plummer ${ }^{1,3,5, *(\mathbb{D})}$
}

1 Department of Veterinary Microbiology and Preventative Medicine, College of Veterinary Medicine, Iowa State University, Ames, IA 50011, USA; afbeyi@iastate.edu (A.F.B.); dgoulart@iastate.edu (D.B.-G.); bruddell@iastate.edu (B.R.); zhang123@iastate.edu (Q.Z.)

2 College of Veterinary Medicine, Iowa State University, Ames, IA 50011, USA; tjh3@iastate.edu (T.H.); clareslagel@gmail.com (C.S.)

3 Department of Veterinary Diagnostic and Production Animal Medicine, College of Veterinary Medicine, Iowa State University, Ames, IA 50011, USA; ahassall@iastate.edu (A.H.); gdewell@iastate.edu (G.D.); osahin@iastate.edu (O.S.)

4 Center for Food Security and Public Health, College of Veterinary Medicine, Iowa State University, Ames, IA 50011, USA; rdewell@iastate.edu

5 National Institute of Antimicrobial Resistance Research and Education, Iowa State University, Ames, IA 50010, USA

* Correspondence: pplummer@iastate.edu

\section{check for} updates

Citation: Beyi, A.F.; Brito-Goulart, D.; Hawbecker, T.; Slagel, C.; Ruddell,

B.; Hassall, A.; Dewell, R.; Dewell, G.;

Sahin, O.; Zhang, Q.; et al.

Danofloxacin Treatment Alters the Diversity and Resistome Profile of Gut Microbiota in Calves. Microorganisms 2021, 9, 2023. https:// doi.org/10.3390/microorganisms9102023

Academic Editor: Steven. P. Djordjevic

Received: 8 September 2021

Accepted: 23 September 2021

Published: 24 September 2021

Publisher's Note: MDPI stays neutral with regard to jurisdictional claims in published maps and institutional affiliations.

Copyright: (c) 2021 by the authors. Licensee MDPI, Basel, Switzerland. This article is an open access article distributed under the terms and conditions of the Creative Commons Attribution (CC BY) license (https:// creativecommons.org/licenses/by/ $4.0 /)$.

\begin{abstract}
Fluoroquinolones, such as danofloxacin, are used to control bovine respiratory disease complex in beef cattle; however, little is known about their effects on gut microbiota and resistome. The objectives were to evaluate the effect of subcutaneously administered danofloxacin on gut microbiota and resistome, and the composition of Campylobacter in calves. Twenty calves were injected with a single dose of danofloxacin, and ten calves were kept as a control. The effects of danofloxacin on microbiota and the resistome were assessed using $16 \mathrm{~S}$ rRNA sequencing, quantitative real-time PCR, and metagenomic Hi-C ProxiMeta. Alpha and beta diversities were significantly different $(p<0.05)$ between pre-and post-treatment samples, and the compositions of several bacterial taxa shifted. The patterns of association between the compositions of Campylobacter and other genera were affected by danofloxacin. Antimicrobial resistance genes (ARGs) conferring resistance to five antibiotics were identified with their respective reservoirs. Following the treatment, some ARGs (e.g., ant 9 , tet 40 , tet $\mathrm{W}$ ) increased in frequencies and host ranges, suggesting initiation of horizontal gene transfer, and new ARGs (aac6, erm $\mathrm{F}$, tet $\mathrm{L}$, tet $\mathrm{X}$ ) were detected in the post-treatment samples. In conclusion, danofloxacin induced alterations of gut microbiota and selection and enrichment of resistance genes even against antibiotics that are unrelated to danofloxacin.
\end{abstract}

Keywords: antimicrobial resistance genes (ARGs); bovine calves; bovine respiratory disease; Campylobacter; danofloxacin/fluoroquinolones; fecal microbiota; resistome

\section{Introduction}

The rise in the incidence of antimicrobial resistance has become the intractable challenge of public health in the 21st century [1]. Use of antimicrobials in the agricultural sector, especially in the livestock industries, has been identified as a contributor toward the acceleration of the selection and spread of antimicrobial-resistant bacterial strains. This makes livestock farms a potential source of resistant strains and antimicrobial resistance genes (ARGs) [2-4]. Gut microbiota is considered an additional "organ" for animals because of its beneficial effects on the physiology, metabolism, immunology, digestion, and nutrient uptake of the host $[5,6]$. However, it can also harbor ARGs, which may spread to humans through the food chain or environmental contamination $[1,4,7,8]$. This situation demands a clear understanding of the variables involved in the emergence and maintenance of 
antimicrobial-resistant bacterial strains and genes in livestock farms and a "One Health" approach to curb their spread to environments and humans.

Fluoroquinolone drugs, such as danofloxacin and enrofloxacin, are frequently used to prevent and control the Bovine Respiratory Disease (BRD) complex in U.S. cattle populations [9-11]. BRD is the most significant disease of young cattle in North America. Furthermore, it accounts for $70 \%$ to $80 \%$ of all feedlot morbidity and $40 \%$ to $50 \%$ of all mortality in the U.S. feedlots [11]. In 2011, a feedlot survey conducted by the National Animal Health Monitoring System (NAHMS) estimated 16.2\% of cattle placed in feedlots showed signs of respiratory disease at some point during their stay on feedlots. Most of these animals $(87.5 \%)$ were treated with antibiotics, of which $99.0 \%$ of the treated calves were injected with antibiotics [12]. Metaphylaxis, mass medication to reduce the incidence of a disease in a group of animals, has also been a well-documented, widely practiced strategy to decrease morbidity and mortality associated with BRD in high-risk cattle [11]. A survey conducted on feedlots by NAHMS in 2011 estimated that $21.3 \%$ of all animals entering the feedlot underwent metaphylaxis [12]. BRD is a significant health problem for dairy cattle as well. In 2007, NAHMS surveyed dairies in 17 states and estimated that $12.4 \%$ of pre-weaned heifer calves had been diagnosed with respiratory disease, and $5.5 \%$ were administered antibiotics $[13,14]$. BRD incidence has demonstrated seasonal variations and is typically increased in the fall season, which often coincides with the shipping of calves from their farm/ranch origin to the feedlot as well as inclement weather [15]. The Food and Drug Administration (FDA) reports that sales and distribution of fluoroquinolones as well as other medically important drugs have increased in recent years [16]. Fluoroquinolones, used for the control of BRD and individual animal treatment, accounted for $1.8 \%$ of the total regimens of antimicrobial drugs used in 22 U.S. beef feedyards in the years 2016 and 2017, which was moderately high compared to some other antimicrobials used for the same purposes (ranging from $0.11 \%$ (sulfonamide) to $9.9 \%$ (macrolide)) [17].

Ciprofloxacin, a fluoroquinolone antibiotic with the same principal agent as that of animal formulations, is commonly used to treat various human infections, including gastroenteritis caused by Campylobacter infection [18,19]. The increased incidence of ciprofloxacin resistance among foodborne pathogens has become a serious threat to public health, which has led to the first removal of fluoroquinolones from use on poultry farms in the U.S. in 2005 [20]. Despite the withdrawal, the trend of fluoroquinolone resistance in Campylobacter species has continued to increase in the last two decades (https://www.cdc.gov/DrugResistance/Biggest-Threats.html\#cam, accessed on $14 \mathrm{Au}$ gust 2020). It has been suggested that the rise in ciprofloxacin resistance in Campylobacter in humans may, at least in part, be associated with use of danofloxacin and enrofloxacin in other livestock [21,22]. Beyond inducing antibiotic resistance in specific bacterial pathogens, these antibiotics exert selection pressure on gut microbiota that may lead to its alteration and enhance the spread of ARGs within bacterial communities [23-25].

Perturbation of gut microbiota following exposure to antibiotics has been a wellrecorded phenomenon in monogastric animals, including humans [26-31]. However, only limited information is available in cattle, and the available data demonstrate inconsistent results and conclusions among studies. In one study, intramuscularly administered penicillin modified the structure of fecal microbiota in calves [32]. Conversely, another group of researchers reported only minor alterations in community structure and the absence of changes in fecal microbial diversity in steers that received single and multiple doses of enrofloxacin subcutaneously [33]. In agreement with the 2018 study, comparisons of the fecal microbiota of calves with and without a history of antimicrobial exposure on beef farms were not different [34]. However, E. coli, used as an indicator organism for the impacts of antibiotics on gut bacteria, showed transient resistance (i.e., a temporary increase in minimum inhibitory concentration) [33], and increased resistance to multiple unrelated antimicrobial agents [32]. In general, the inconsistency among the studies could partly be attributed to differences in their study designs and methods as well as the inherent complexity within the gut microbiota [35]. 
The objective of this study was to assess the impacts of danofloxacin administered subcutaneously to bovine calves on the fecal microbiota, Campylobacter, and resistome (i.e., all detectable ARGs within a given sample). We hypothesized that infusion of danofloxacin and its metabolite, ciprofloxacin, from plasma into the intestine following a single-dose subcutaneous injection of danofloxacin will exert selective pressure on gut microbiota and alter its diversity and the resistome profile. In this study, we sequenced the 16S rRNA gene to compare the compositions and structures of fecal microbiota before and after the treatment and to assess changes in the compositions of Campylobacter. Additionally, we employed a novel proximity ligation-guided metagenomics approach (ProxiMeta) that has the advantage of linking the ARGs with their microbial reservoirs over other metagenomic methods [36], which enabled us to track changes in antibiotic-resistant determinants as driven by danofloxacin administration. Quantitative real-time PCR (qPCR) was employed to assess the impacts of danofloxacin on the dynamics of selected ARGs.

\section{Results}

\subsection{Results of $16 S$ rRNA Gene Analysis}

The total number of reads in our samples was $10,999,180$, with an average of 52,377 reads per sample (SD 12,721, range 5179-124,738, and median 51,698 reads per sample). A total of 6628 bacterial Operational Taxonomic Units (OTUs) were identified in 210 samples, which were classified into 298 genera (mean $=91$ genera, $\mathrm{SD}=7$, and range $=68-116$ per sample), 35 classes (mean $=23$ classes, $\mathrm{SD}=2$, and range $=16-28$ per sample), and 19 phyla (mean $=13$ phyla, $\mathrm{SD}=1$, and range $=9-17)$. A random subsample of 11,500 reads per sample was used to normalize sequence numbers for the computation of alpha and beta diversity metrics; one sample with 5179 reads was excluded from the analyses due to insufficient depth.

\subsubsection{Bacterial Phyla by Sampling Days}

The relative abundances of phyla detected in this study were compared by the sampling days (Supplementary Materials Table S1). Phyla with the highest relative abundance in the first sampling day, two days after the calves arrived at the animal study facility, included Firmicutes (relative abundance $=51.93 \%)$, Bacteroidetes $(36.13 \%)$, Proteobacteria $(3.00 \%)$, Tenericutes $(2.18 \%)$, Spirochaetes (1.94\%), Actinobacteria $(1.33 \%)$, and Verrucomicrobia $(1.03 \%)$. The relative abundances of the phyla varied over the subsequent sampling days during the four weeks of the study period; however, Firmicutes and Bacteroidetes remained dominant phyla throughout that time.

\subsubsection{Alpha Diversities}

The sequencing depth was evaluated using alpha rarefaction curves (figures not shown); for all considered alpha diversity metrics, the sequencing depth was sufficient to reveal rare microbes in the samples. Calves in group $C$ that were challenged with Mannheimia haemolytica exhibited mild to moderate signs of BRD. However, microbial compositions and diversities were not significantly different from group $B$, the group that was not challenged with $M$. haemolytica but injected with danofloxacin subcutaneously. Thus, these two groups that received danofloxacin were collapsed together into one group, named the treatment group, for the comparison of microbial evenness and richness before and after the treatment. Based on the analyses, observed OTUs measuring the microbiota richness showed a significant change between pre- and post-treatment samples $(p<0.05)$. Similarly, microbiota evenness measured by the Shannon index was different between pre- and post-treatment samples ( $p<0.05$, Figure 1$)$. Strikingly, both microbial richness and evenness significantly increased following danofloxacin administration, as shown in Figure 1. 

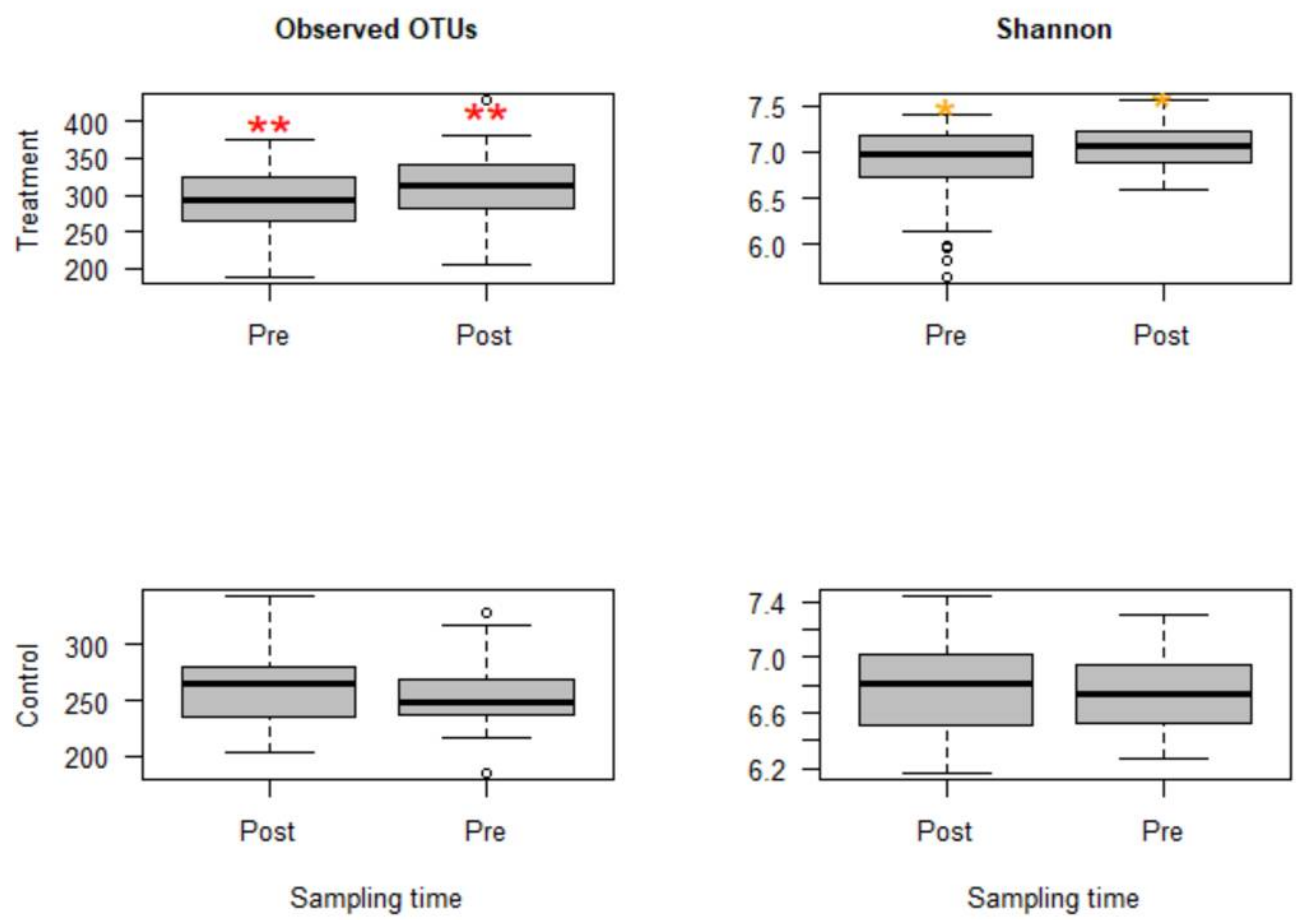

Figure 1. Boxplots for alpha diversity metrics comparing pre- and post-treatment samples in the treatment group (top) and control group (bottom). The treatment group received a single subcutaneous injection of danofloxacin. Asterisks show significant differences $\left({ }^{*} p<0.05,{ }^{* *} p<0.01\right)$ between pre- and post-treatment microbial communities for the respective group. Note: the control group was divided into pre- and post-treatment to compare with the treatment group in a temporal manner; otherwise, the calves in the control group did not receive any antibiotic. The observed OTUs and Shannon index do not have units, but they measure microbial richness and evenness based on OTU counts and abundance.

Since the fecal samples were collected from the study calves over 28 days, there was an anticipation that the fecal microbiota would change over time because the calves were around two months old at the beginning of the study, the age at which microbial diversity and structure alter fast. Thus, linear mixed-effects models were run using QIIME2 to discriminate between microbial changes attributed to age from those due to danofloxacin exposure. Accordingly, the effect of time was not significant for either measure in the control group. In contrast, the time had a marginally significant influence (observed OTUs, $p=0.064$ and Shannon, $p=0.091$ ) in the treatment group, indicating the influence of danofloxacin on the microbial compositions and diversities was more prominent than the impact of the time in this study.

\subsubsection{Beta Diversities}

Beta-diversity was measured by the Bray-Curtis dissimilarity index, which can be visualized in the NMDS plot displayed in Figure 2. Gut microbiota shifted after the treatment, as shown in the figure by a clustering difference between pre- and post-treatment microbial communities, and the community structures were significantly different $(p<0.05)$. 


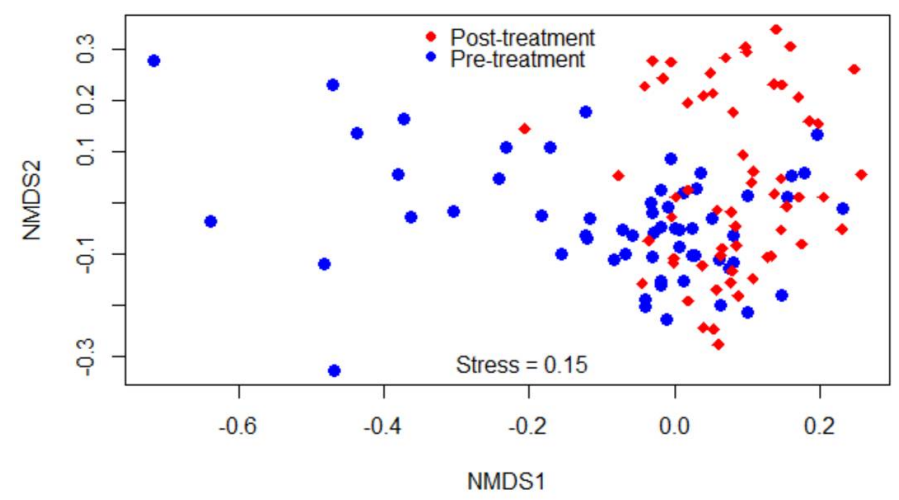

Figure 2. Non-metric multidimensional scaling (NMDS) of the Bray-Curtis dissimilarity index showing clustering difference between pre- and post-treatment microbial communities, blue and red dots, respectively.

\subsubsection{Comparisons of Compositions of Bacterial Classes among Study Calf Groups}

The relative abundances of bacterial taxa were compared among the calf groups using the Kruskal-Wallis and pairwise Dunn tests (Table S2). For many of the bacterial classes, a significant difference $(p<0.05)$ was observed between the control group (Group A) and the treatment groups (Group B or C, or both). For instance, Methanobacteria, Thermoplasmata, Betaproteobacteria, Epsilonproteobacteria, and Mollicutes significantly increased in the treatment groups, whereas Bacilli, Planctomycetia, and Synergistia decreased. Some bacterial classes, such as Bacteroidia, Cyanobacteria-4C0d-2, and Clostridia did not show significant changes. Comparisons between the treatment groups, which were BRD-induced Group C against non-BRD-induced Group B, had shown significant differences for certain bacterial classes such as Actinobacteria, Planctomycetia, Betaproteobacteria, and Tenericutes- RF3.

2.1.5. Comparisons of Relative Abundance of Bacterial Genera between Pre- and Post-Treatment Communities

After the classification of the reads at a minimum of $97 \%$ similarity into OTUs using QIIME 1.9, a total of 4770 OTUs were obtained. Two hundred and sixty-five of the OTUs in the treatment group increased, and 211 of them decreased after danofloxacin injection at FDR $p \leq 0.01$ (379 and 319 at FDR $p \leq 0.05$, respectively). Furthermore, the relative abundance of genera in pre- and post-treatment samples were compared using the Wilcoxon signed-rank test and Boruta algorithm/random forest models. Out of 228 genera with relative abundance $\geq 0.01 \%$, 66 of them had significantly shifted after the treatment as indicated by the Wilcoxon signed-rank test $(p<0.05$, Table 1$)$ and 57 genera after the $p$-value was adjusted by FDR $(p<0.05)$. These genera fell into 12 phyla, with 31 of them in Firmicutes, 13 in Proteobacteria, 8 in Bacteroidetes, 3 in Actinobacteria, 3 in Spirochaetes, 2 in Elusimicrobia, and the rest in the other six phyla. 
Table 1. Comparisons of the relative abundance of bacterial genera between fecal samples collected before and after danofloxacin injection in the treatment group by the Wilcoxon signed-rank test. Only significantly altered genera were shown in the table.

\begin{tabular}{|c|c|c|c|c|c|c|c|}
\hline \multirow{2}{*}{ Genus } & \multicolumn{2}{|c|}{ Relative Abundance (\%) } & \multirow{2}{*}{$p$} & \multirow{2}{*}{ Genus } & \multicolumn{2}{|c|}{ Relative Abundance (\%) } & \multirow{2}{*}{$p$} \\
\hline & Pre & Post & & & Pre & Post & \\
\hline Succinivibrio & 0.38 & 0.05 & 0.000 & Erysipelotrichales_RFN20 & 0.04 & 0.03 & 0.000 \\
\hline Streptophyta_5-7N15 & 4.12 & 8.31 & 0.000 & Desulfovibrio & 0.51 & 0.27 & 0.000 \\
\hline Enterobacteriaceae_unclassified & 0.08 & 0.01 & 0.000 & Elusimicrobiaceae_unclassified & 0.12 & 0.04 & 0.000 \\
\hline Peptococcaceae_unclassified & 0.13 & 0.25 & 0.000 & Bacteroidaceae_unclassified & 1.53 & 2.54 & 0.000 \\
\hline Alphaproteobacteria_unclassified & 0.38 & 0.14 & 0.000 & Synergistes & 0.03 & 0.01 & 0.000 \\
\hline Eubacterium & 0.13 & 0.03 & 0.000 & Methanosphaera & 0.35 & 0.22 & 0.001 \\
\hline Coprobacillus & 0.01 & 0.00 & 0.000 & Sutterella & 0.61 & 0.24 & 0.001 \\
\hline Erysipelotrichales_p-75-a5 & 0.10 & 0.00 & 0.000 & Pseudoramibacter & 0.05 & 0.02 & 0.001 \\
\hline Prevotella & 2.29 & 0.93 & 0.000 & Rikenellaceae_unclassified & 5.57 & 3.44 & 0.003 \\
\hline Blautia & 0.27 & 0.05 & 0.000 & Epulopiscium & 0.04 & 0.08 & 0.004 \\
\hline [Paraprevotellaceae]_unclassified & 0.04 & 0.00 & 0.000 & Treponema & 1.98 & 1.45 & 0.005 \\
\hline Faecalibacterium & 0.15 & 0.00 & 0.000 & Clostridiales_unclassified & 9.72 & 8.65 & 0.005 \\
\hline Phascolarctobacterium & 0.51 & 0.36 & 0.000 & [Barnesiellaceae]_unclassified & 0.19 & 0.32 & 0.008 \\
\hline Anaerovibrio & 0.10 & 0.03 & 0.000 & Bifidobacterium & 0.37 & 0.12 & 0.013 \\
\hline Mogibacteriaceae_unclassified & 0.69 & 0.50 & 0.000 & Desulfovibrionaceae_unclassified & 0.20 & 0.28 & 0.014 \\
\hline Butyrivibrio & 0.09 & 0.16 & 0.000 & Akkermansia & 2.94 & 4.72 & 0.018 \\
\hline Cyanobacteria_unclassified & 1.16 & 0.75 & 0.000 & Elusimicrobium & 0.22 & 0.29 & 0.022 \\
\hline Veillonellaceae_unclassified & 0.18 & 0.13 & 0.000 & Peptostreptococcaceae_unclassified & 1.61 & 2.33 & 0.023 \\
\hline Ruminococcaceae_unclassified & 27.17 & 31.87 & 0.000 & Bacteroides & 1.83 & 0.23 & 0.042 \\
\hline Pirellulaceae_unclassified & 0.05 & 0.14 & 0.000 & Ruminobacter & 0.01 & 0.02 & 0.046 \\
\hline
\end{tabular}

For the classification of the microbial communities into pre- and post-treatment, out of 298 genera, 44 of them were confirmed as important attributes by the Boruta algorithm, meaning the treatment affected the proportions of these genera significantly. The feeding of these important genera to the random forest model resulted in an out of bag error of $2.06 \%$, accuracy for the training sub-data of 100\% (95\% CI: 96.27-100\%), and an accuracy rate for the test sub-data of 100\% (95\% CI: 91.59-100\%). These 44 genera, classifying the microbial communities into pre- and post-treatment communities, were ranked by the random forest model based on their importance, as depicted in Figure 3. Four Proteobacteria genera (Succinivibrio, RF32_g_unclassified, Gallibacterium, Enterobacteriaceae_g_unclassified) and Bacteriaodetes_g_5.7N15 were the most important. Interestingly, 37 genera were identified as having a significant association with or predictors of pre/post-treatment status by both the Wilcoxon signed-rank test and the Boruta/random forest method.

Furthermore, the number of genera per sample $24 \mathrm{~h}$ after the treatment was significantly lower $(p<0.05)$ in the treatment group than in the control group (Figure 4$)$. For groups B and C, the counts were significantly reduced at $24 \mathrm{~h}$ after the treatment compared with one day earlier sampling or four or seven days after the treatment (i.e., sampling days 19 vs. 18,19 vs. 22,19 vs. $28, p<0.05$; day 18 was the treatment day). In contrast, none of them were significant in the control group. 


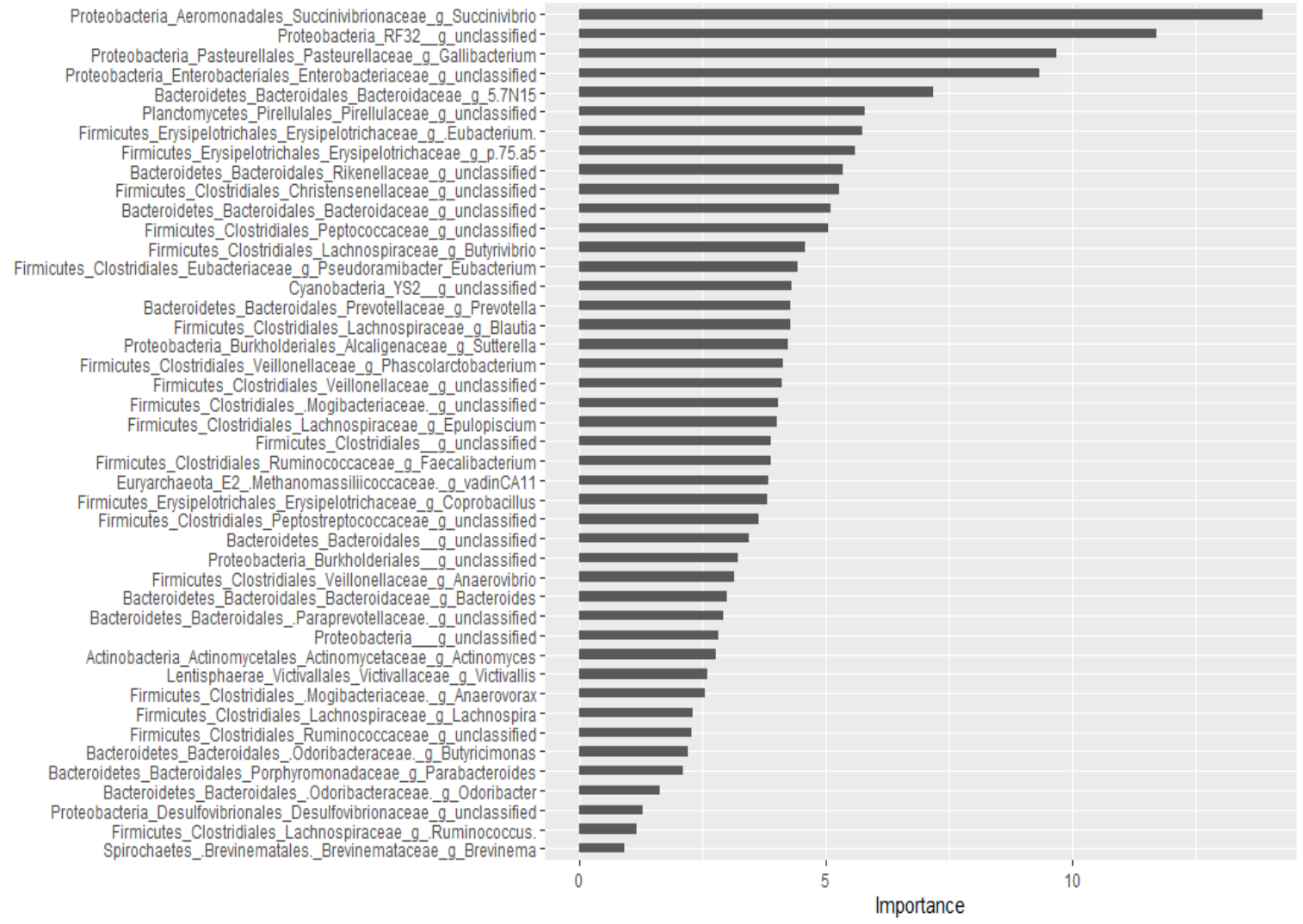

Figure 3. Bacterial genera that classify pre- and post-treatment communities based on the Boruta important variable selection algorithm and ranking by the random forest model. The length of the horizontal bars corresponds to its predictive importance.

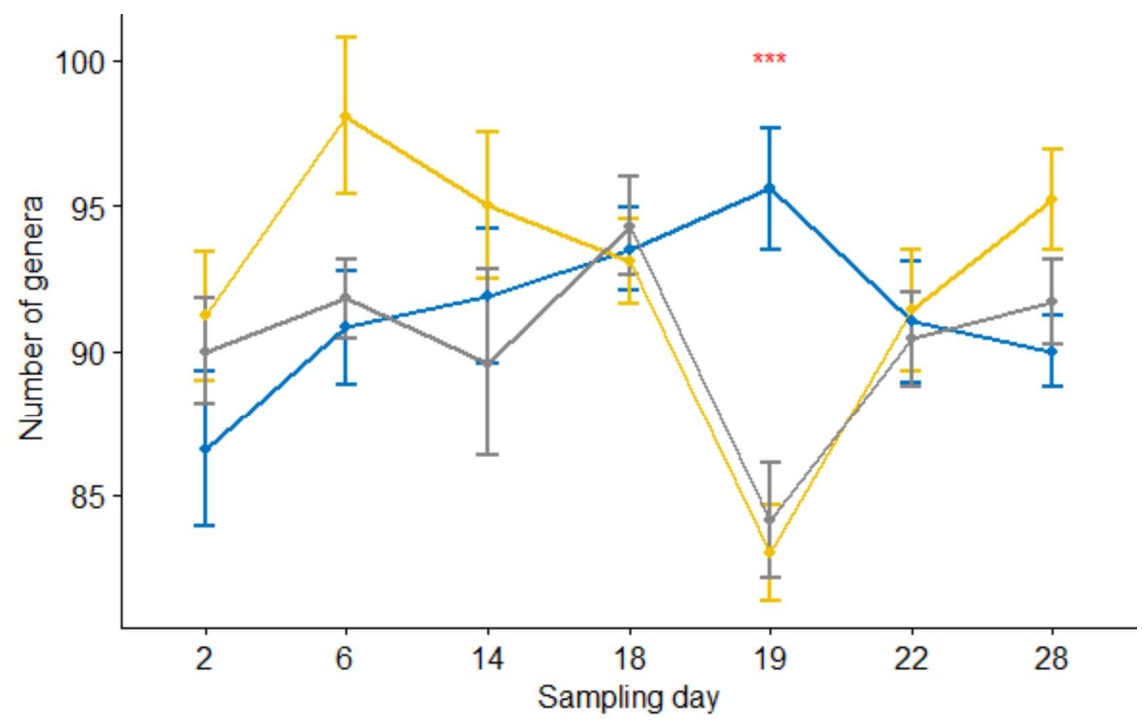

Group $\rightarrow A \rightarrow B \rightarrow C$

Figure 4. Number of genera per sample for three calf groups by sampling time point. Genus count at sampling Day 19 were significantly lower in both treatment groups B and C than in the control group A. C. jejuni cocktail, M. haemolytica, and danofloxacin were administered on Days 4, 10 and 18, respectively. ${ }^{* * *} p=0.001$. 


\subsection{The Compositions of Campylobacter}

\subsubsection{Comparison by Sampling Days}

The relative abundances of Campylobacter in samples collected on Day 2 through Day 28 were $0.03 \%, 0.03 \%, 0.35 \%, 0.72 \%, 0.04 \%, 0.31 \%$, and $0.76 \%$ in the pooled data of the combined treatment groups (groups C and B), respectively. Its composition on Day 19 (24 $\mathrm{h}$ after danofloxacin administration) was significantly lower than the composition on Day 18, as well as than those on the last two sampling days. Figure 5 depicts the proportions of Campylobacter in the samples collected at seven sampling times compared by the Kruskal-Wallis non-parametric and its post hoc tests. Campylobacter level dropped sharply and significantly $24 \mathrm{~h}$ after the treatment; however, it returned to the pre-treatment baseline after three days (i.e., no significant difference between the proportions on Day 18 and Day 22, $p>0.05$ ). In contrast, the proportions of Campylobacter did not show significant variations among sampling days except for a slight rise in the last sampling day in the control group (results not shown).

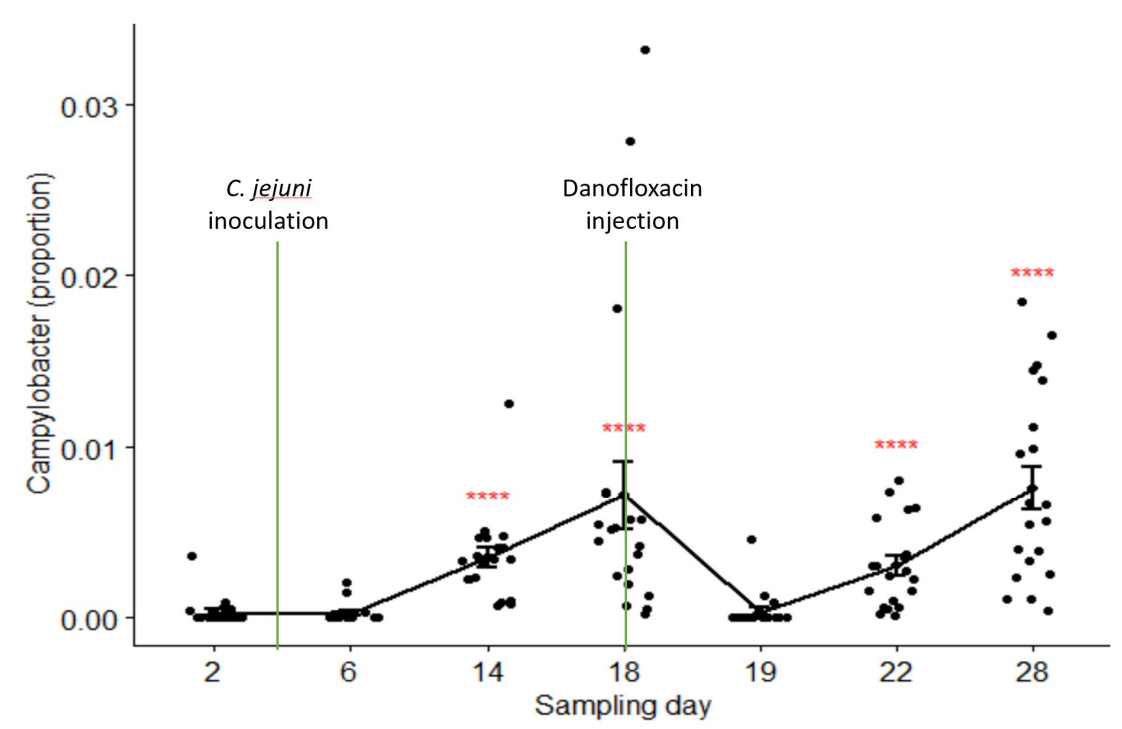

Figure 5. Comparisons of the relative abundance of Campylobacter in samples collected from the calves in the combined treatment groups (B and C) by sampling days. Danofloxacin was injected on Day 18 right after fecal sample collection. ${ }^{* * *}$ relative abundance of Campylobacter on Day 19 was significantly different from those on the sampling Day 14, Day 18, Day 22, and Day 28. The abundance rates were significantly different from each other on sampling Day 22 and Day $28(p=0.000)$.

\subsubsection{Correlation with Other Genera}

The relative abundance of Campylobacter was compared with other genera using the Spearman rank correlation test (Tables S3 and S4). Twenty-one genera had a significant correlation with Campylobacter in the pooled data (all groups combined), of which ten of them had a negative correlation. Seven of the eight Bacteroidetes genera that significantly correlated with Campylobacter had negative correlation coefficients. In contrast, most of the genera in Firmicutes and Proteobacteria had a positive association with the relative abundance of Campylobacter.

On the other hand, pre- and post-treatment data were separately analyzed to assess if the treatment had an effect on the pattern of correlation between Campylobacter and other genera. Seven and eleven genera in pre- and post-treatment samples had a significant correlation with Campylobacter, respectively. Interestingly, pre- and post-treatment samples shared only one genus in common that had a significant association with Campylobacter, which was an unclassified genus in the family of Desulfovibrionaceae. This result suggests that danofloxacin exposure altered the correlation pattern between bacterial taxa. 


\subsubsection{Prediction of Important Genera by Random Forests}

Using the Boruta algorithm, out of 298 genera, nine of them were confirmed as important attributes in the combined data of all groups. Based on the random forest model, these nine genera explained $46.01 \%$ of the variability in the relative abundance of Campylobacter with a very low mean squared error (i.e., 0.059), indicating the best model fitness. Except for two, these genera had significant Spearman rank correlation coefficients as well. Furthermore, genera ranked as very important by the random forest model had the highest Spearman correlation coefficients. Additional analyses were performed by dividing data as pre-treatment, post-treatment, and control to check if the treatment would have changed the association patterns between genera. In agreement with the Spearman rank correlation test, there was a difference between pre- and post-treatment samples in both the types and numbers of genera correlated with the compositions of Campylobacter in the fecal samples. Figure 6 presents genera that predict the relative abundance of Campylobacter in the total, the pre- and post-treatment, and the control samples separately.
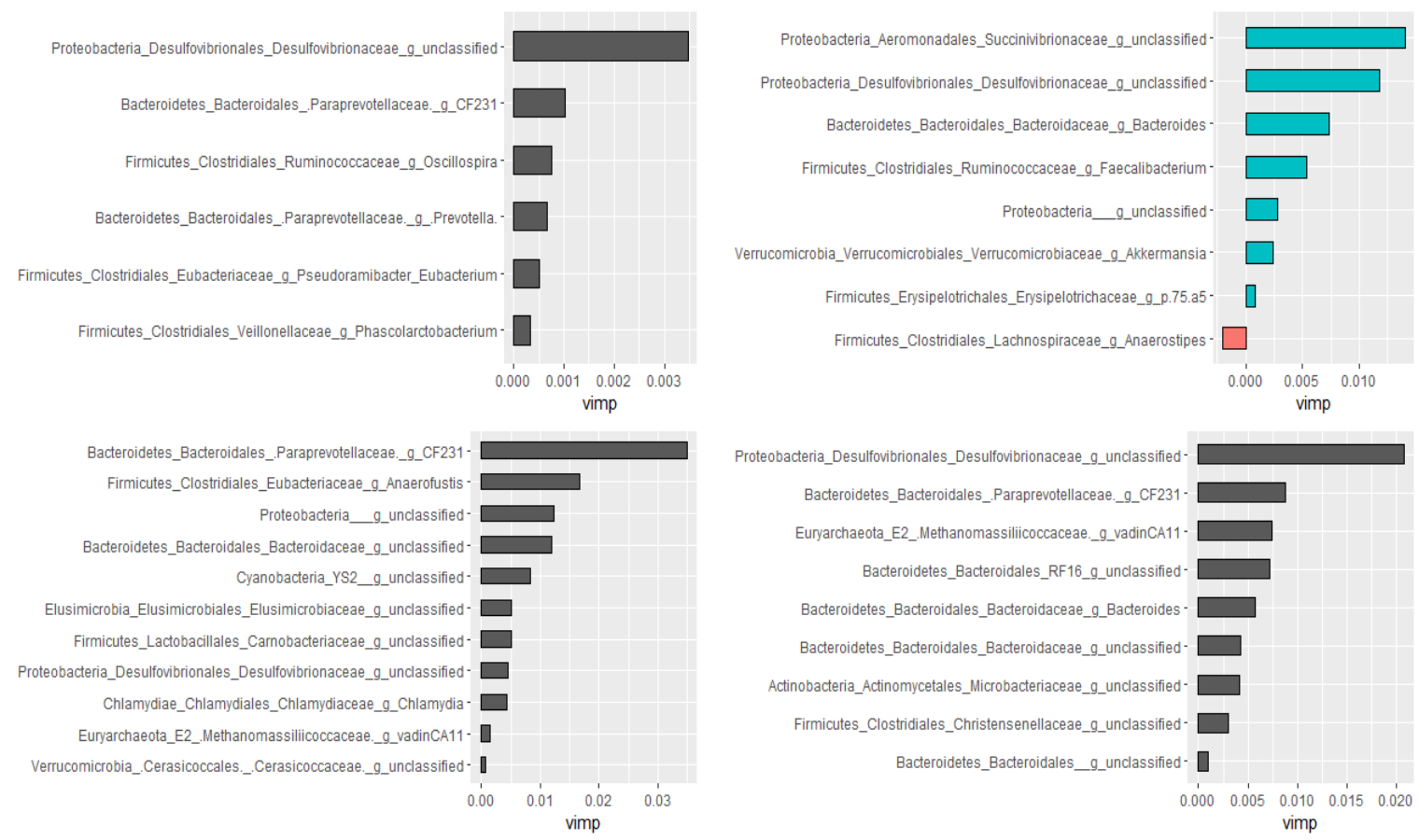

Figure 6. Genera associated with the relative abundance of Campylobacter selected by the Boruta algorithm and ranked by the random forest models. Control (top left), pre-treatment (top right), post-treatment (bottom left), and all data (bottom right). The length of the bar is proportional to the predictive importance of the genera; vimp-variable importance.

\subsection{Results of Metagenomic Hi-C (ProxiMeta)}

To assess the influence of danofloxacin injection on fecal resistome profiles, pooled samples from pre- and post-treatment samples were sequenced using metagenomic HiC. Accordingly, genes conferring resistance to tetracycline, aminoglycosides, $\beta$-lactams, macrolides, and phenicol were detected in both pre- and post-treatment samples (Table 2). The abundance of tetracycline resistance determinants was the highest with eight and nine different ARG types in pre-and post-treatment pooled samples, respectively. tet $\mathrm{Q}$, tet $\mathrm{W}$, and tet 40 showed a dramatic increase both in number of hits and host ranges following the treatment; tet $\mathrm{B}$ was detected only in the pre-treatment sample, while tet $\mathrm{L}$ and tet $\mathrm{X}$ were identified after the treatment samples only. tet 32 and tet $\mathrm{O}$ were carried on the same contig. Six unique aminoglycoside ARG types were detected in the samples with aac6 only observed in the post-treatment sample. The number of ant 9 copies increased dramatically following the treatment from 39 to 189 with host range increase from 12 to 23 . The rest of the aminoglycoside ARGs showed minor variations between pre-and post-treatment 
pooled samples. Two or more ARG types were carried on the same contig. Four unique macrolide ARGs were identified, ermB being undetected in the post-treatment sample, while the rest of them did not fluctuate both in copy numbers and host ranges between the two sampling time points. aci and $c f X \beta$-lactam ARGs were reported in this study with the latter ARG emerged after the treatment. Only one phenicol resistance gene ( $c f r$ ) was observed, which significantly increased both in the number of hits and reservoirs following the treatment.

Table 2. Summary of antimicrobial resistance genes in pooled fecal samples before and after danofloxacin injection in calves identified by Metagenomic Hi-C.

\begin{tabular}{|c|c|c|c|c|c|}
\hline \multirow[t]{2}{*}{ Antibiotic Class } & \multirow[t]{2}{*}{ Resistance Gene } & \multicolumn{2}{|c|}{ Number of Hits } & \multicolumn{2}{|c|}{ Number of Hosts } \\
\hline & & $\begin{array}{c}\text { Pre-trt } \\
\left(165^{*}, 38^{* *}\right)\end{array}$ & $\begin{array}{c}\text { Post-trt } \\
(200,64)\end{array}$ & $\begin{array}{c}\text { Pre-trt } \\
(165,38)\end{array}$ & $\begin{array}{r}\text { Post-trt } \\
(200,64)\end{array}$ \\
\hline \multirow[t]{6}{*}{ Aminoglycoside } & aac6 & 0 & 3 & 0 & 3 \\
\hline & aph2 & 61 & 58 & 14 & 16 \\
\hline & aph3 & 51 & 55 & 11 & 13 \\
\hline & ant6 & 75 & 84 & 22 & 29 \\
\hline & ant9 & 39 & 189 & 12 & 23 \\
\hline & sat & 51 & 56 & 11 & 12 \\
\hline \multirow[t]{2}{*}{ Beta-lactams } & $a c i$ & 1 & 4 & 1 & 2 \\
\hline & $c f X$ & 5 & 8 & 5 & 3 \\
\hline \multirow[t]{5}{*}{ Macrolides } & erm $\mathrm{B}$ & 8 & 0 & 3 & 0 \\
\hline & erm $\mathrm{F}$ & 0 & 1 & 0 & 1 \\
\hline & erm $\mathrm{G}$ & 24 & 5 & 3 & 3 \\
\hline & ermQ & 1 & 0 & 1 & 0 \\
\hline & $m e f \mathrm{E}$ & 3 & 1 & 2 & 1 \\
\hline Phenicol & $c f \mathrm{R}$ & 15 & 24 & 3 & 14 \\
\hline \multirow[t]{10}{*}{ Tetracyclines } & tet32 & 20 & 16 & 9 & 7 \\
\hline & tet40 & 112 & 1484 & 44 & 91 \\
\hline & tet 44 & 15 & 6 & 10 & 5 \\
\hline & tet $\mathrm{A}$ & 8 & 4 & 5 & 3 \\
\hline & tet $\mathrm{B}$ & 1 & 0 & 1 & 0 \\
\hline & tet $\mathrm{L}$ & 0 & 69 & 0 & 5 \\
\hline & tet $\mathrm{O}$ & 78 & 75 & 27 & 21 \\
\hline & tetQ & 25 & 483 & 10 & 19 \\
\hline & tetW & 178 & 2836 & 79 & 105 \\
\hline & tetX & 0 & 1 & 0 & 1 \\
\hline
\end{tabular}

${ }^{a}$ Pre-treatment samples, pooled individual samples collected right before danofloxacin injection (Day 18); ${ }^{\mathrm{b}}$ Post-treatment samples, pooled individual samples collected four days after danofloxacin injection (Day 22). * Total number of clusters in the pooled samples, and ${ }^{* *}$ number of clusters with $\geq 80 \%$ complete genomes.

Using proximity ligation, the linkage of ARGs with their respective bacterial hosts was also established in the present study. Phyla associated with the current ARGs are presented in Table 3. Firmicutes was the leading phylum associated with these ARGs, whereas Bacteroidetes appeared to acquire the ARGs following the treatment. At lower bacterial classification level, most of these resistance genes were hosted by class Clostridium species, Eubacterium species, and Sharpea azabuensis DSM_18934 in the pre-treatment samples, and Clostridium, Eubacterium, Prevotella, and Ruminococcus species in the post-treatment samples (Table S5). Most of the identified ARGs were carried by more than one clusters of bacterial taxa having $\geq 80 \%$ complete genomes; for instance, tet $\mathrm{W}$ was detected in 12 and 49 clusters in pre-and post-treatment samples, indicating a wide range of distribution. Table S5 presents copy numbers and bacterial taxa (at a lower classification level) associated with each ARG. 
Table 3. Summary of phyla associated with antimicrobial resistance genes detected in pooled fecal samples before and after danofloxacin administration.

\begin{tabular}{|c|c|c|}
\hline Phylum & Pre-Treatment & Post-Treatment \\
\hline Actinobacteria & ermB, ermG, tetW & ant 9 , tet 40 , tetQ, tetW \\
\hline Bacteroidetes & tetQ & $\begin{array}{l}\text { aph2, aph3, ant6, cfX, ermF, ermG, } \\
\text { mefE, cfR, tet40, tetQ, tetW }\end{array}$ \\
\hline Euryarchaeota & ant 9 , tet $\mathrm{A}$, tetO & No ARGs \\
\hline Firmicutes & $\begin{array}{l}\text { aph2, aph3, ant6, ant } 9 \text {, sat, } \operatorname{cfX} \text {, ermB, } \\
\text { cfR, tet } 32 \text {, tet } 40 \text {, tet } 44 \text {, tetA, tetB, tetO, } \\
\text { tetQ, tetW }\end{array}$ & $\begin{array}{l}\text { aac6, aph2, aph3, ant6, ant } 9 \text {, sat, } \\
\text { ermG, cfR, tet32, tet } 40 \text {, tetA, tetL, } \\
\text { tetO, tetQ, tetW }\end{array}$ \\
\hline Proteobacteria & $\begin{array}{l}\text { aph2, aph3, ant6, ant9, sat, tet32, tet } 40 \text {, } \\
\text { tetO, tetW }\end{array}$ & $\begin{array}{l}\text { aac6, aph2, aph3, ant6, ant9, cfR, } \\
\text { tet40, tetL, tetQ, tetW }\end{array}$ \\
\hline Spirochaetes & NA & ant6, sat, tet 40 , tet 44 , tetW \\
\hline Tenericutes & tet40 & No ARGs \\
\hline Verrucomicrobia & tet40 & ant6, sat, tet 40 , tetW \\
\hline
\end{tabular}

\subsection{Results of $q P C R$}

To validate the Hi-C results, a subset of genes was selected for qPCR testing. Primers targeting tet $\mathrm{W}$, tet $\mathrm{O}$, tet $\mathrm{X}$, erm $\mathrm{B}$, and erm $\mathrm{F}$ were used to compare the changes in their quantity following danofloxacin administration. Comparison of the treatment groups (B and C) with the control group using the non-parametric pairwise Dunn test has revealed that tet $\mathrm{W}$ increased significantly (adj. $p<0.05$ ) in the post-treatment pooled samples from group B compared the control. In contrast, tet $\mathrm{O}$ was significantly reduced (adj. $p<0.05$ ) in group $\mathrm{C}$ compared to the control while ermF increased significantly in the same group. The change in the quantity of ermB was significantly lower in group B than the control group (Table 4).

Table 4. Changes in the quantity of selected antimicrobial resistance genes in pooled fecal samples following danofloxacin treatment measured by qPCR.

\begin{tabular}{|c|c|c|c|c|}
\hline & Change & Control & Group B & Group C \\
\hline \multirow[t]{3}{*}{ tetW } & Mean $(\log 2 *)$ & -0.21 & 0.58 & 0.34 \\
\hline & SD & 0.017 & 0.065 & 0.015 \\
\hline & $p$ value ${ }^{* *}$ & NA & $0.034^{\mathrm{a}}$ & 0.272 \\
\hline \multirow[t]{3}{*}{ tet $\mathrm{O}$} & Mean & -0.01 & -0.15 & -0.32 \\
\hline & SD & 0.024 & 0.088 & 0.173 \\
\hline & $p$ value & NA & 0.272 & $0.0338^{c}$ \\
\hline \multirow[t]{3}{*}{ tet $\mathrm{X}$} & Mean & -0.31 & -0.92 & 0.02 \\
\hline & $\mathrm{SD}$ & 0.081 & 0.058 & 0.039 \\
\hline & $p$ value & NA & 0.267 & 0.264 \\
\hline \multirow[t]{3}{*}{ erm B } & Mean & 0.52 & -0.76 & -0.15 \\
\hline & SD & 0.081 & 0.064 & 0.174 \\
\hline & $p$ value & NA & $0.022^{b}$ & 0.359 \\
\hline \multirow[t]{3}{*}{ erm $\mathrm{F}$} & Mean & -0.68 & -0.44 & 0.19 \\
\hline & $\mathrm{SD}$ & 0.054 & 0.095 & 0.173 \\
\hline & $p$ value & NA & 0.359 & $0.022^{d}$ \\
\hline
\end{tabular}

* The values obtained using Gallop method analysis was log2 transformed to compare the fold changes in gene abundance between calf groups. ${ }^{* *}$ An adjusted $p$-value obtained from pairwise comparisons using the Dunn test. ${ }^{\mathrm{a}}$ tet $\mathrm{W}$ increased and ${ }^{\mathrm{b}}$ erm $\mathrm{B}$ decreased significantly in the group B post-treatment pooled sample compared to the control group post-treatment sample. ${ }^{\mathrm{c}}$ tetO decreased and

$\mathrm{d}$ erm $\mathrm{F}$ increased significantly in group $\mathrm{C}$ compared to the control group.

\section{Discussion}

Danofloxacin is frequently administered to beef calves as prophylaxis and metaphylaxis to prevent and control BRD in beef cattle in the U.S. Having a short meat withdrawal time makes it one of the preferred antibiotics used by producers [37]. However, little is known about its unintended side effects on gut microbial compositions, diversities, and resistome. In this study, we investigated the impacts of a single dose of subcutaneously administered danofloxacin on the fecal microbiota, the compositions of Campylobacter, and 
the resistome in calves. Compared to the control group, fecal microbiota was shifted in the treatment groups following danofloxacin injection, as indicated by significant changes in alpha and beta diversities as well as the shift in the relative abundance of bacterial taxa. Additionally, the abundance of Campylobacter has been altered in the treatment groups, likely due to the emergence of antibiotic resistance in this genus (data being prepared under a separate manuscript). Furthermore, our study showed enrichment of antibiotic determinants, which suggests that danofloxacin created an environment that might have favored the dominance of resistant strains and/or transfer of ARGs among bacteria. Given that antibiotic use exerts selective pressure on bacteria for the emergence of resistance, these findings are consistent with evidence by many other studies. The emergence of resistant strains and genes in livestock is worrisome since they shed them with feces and contaminate the farm environments, which may eventually reach the human food chain or drinking water. This necessitates capturing the "One health" paradigm to study AMR and minimizing its economic and public health impacts $[7,8]$.

The effect of danofloxacin on bovine gut microbiota has not been previously studied despite being one of the commonly used fluoroquinolone drugs on beef farms. Fluoroquinolones have a bactericidal effect on bacteria by interfering with the DNA coiling during replication [37]. Their mechanisms involve inhibition of bacterial enzyme topoisomerases, such as topoisomerase II (DNA gyrase) in Gram-negative bacteria and topoisomerase IV in Gram-positive bacteria. Danofloxacin is a third-generation fluoroquinolone drug used for the treatment of respiratory disease in pigs and cattle [38]. Parenteral administration of this drug results in a high concentration in the digestive tract, which increases antibiotic exposures of gut microbiota. According to a study performed on dairy cows, the concentration of danofloxacin in tissue samples from the digestive tract of cattle was high. It was more than five times and 19 times the plasma concentration in the colon and mesenteric lymph nodes at $8 \mathrm{~h}$ and $12 \mathrm{~h}$ post-administration, respectively [38]. The same study indicated a high volume of distribution and prolonged elimination half-life of danofloxacin. Other researchers also demonstrated that marbofloxacin (another fluoroquinolone) doses used to target pathogens in the lungs had a similar effect on the bacterial population in the small intestine [39]. These authors reported 2- to 8-fold higher levels in total marbofloxacin concentrations in the digestive tract than in plasma for over $24 \mathrm{~h}$ following intramuscular injection of this drug to young pigs [39]. However, the bactericidal effect of fluoroquinolones in the intestine is diminished because they are highly bound in the intestines/feces, as demonstrated in pigs [39], rats [40], and humans [41]. Despite the reduced bactericidal effect, available evidence shows that fluoroquinolones create selective pressure in the intestine [18]. One such example is the emergence of fluoroquinolone resistance in Enterobacteriaceae strains following intramuscular injection of enrofloxacin in pigs [42]. The same study revealed that both orally and parenterally administered enrofloxacin induced development of AMR in selected bacterial species with different levels in pigs [42]; however, the difference in the impacts of fluoroquinolone drugs administered via oral and parenteral routes on gut microbiota needs to be investigated in calves. Nevertheless, there is enough evidence from the literature that complements our findings and shows parenterally administered danofloxacin is capable of enhancing selective pressure on gut microbiota and inducing its perturbation.

The major bacterial phyla identified from the fecal samples collected after two days of the beginning of the study (i.e., baseline data) were Firmicutes $(51.93 \%)$, Bacteroidetes $(36.13 \%)$, Proteobacteria $(3.00 \%)$, Tenericutes $(2.18 \%)$, Spirochaetes $(1.94 \%)$, Actinobacteria $(1.33 \%)$, and Verrucomicrobia $(1.03 \%)$. These findings agree with previous studies in beef cattle [43-46]. A study conducted in Canada, however, indicated a variation between beef farms with regard to bacterial dominance; some of the farms were characterized by the dominance of Actinobacteria and Proteobacteria, while the other farms by the dominance of Firmicutes [34]. Several factors, such as diets, antibiotic exposure, genetics, management, and environment, contribute to the variation in microbial compositions and diversities between and within farms $[28,30,47,48]$. In the current study, the calves were originated from 
the same farm, fed the same diets, and managed the same way under similar environment; and none of them had a history of antibiotic exposure prior the study. Thus, microbial variations attributed to these factors were minimal.

In the present study, danofloxacin increased microbial richness (median OTUs rose from 293 to 313) and diversity (median Shannon index rose from 6.98 to 7.08). These findings contrast with our initial hypothesis that the higher concentration of danofloxacin diffusing into the intestine reduces microbial richness and diversity. Consistent with our original hypothesis, a two-week tetracycline administration reduced gut microbial diversity, changed the abundance of some bacterial taxa, and enhanced the abundance of ARGs, plasmids, and integrons in mouse guts [24]. In agreement with our current finding, other authors reported that diversity of microbiota increased in the piglets that received a single injectable dose of tulathromycin compared to the control, which they considered as "more chaotic" [30]. On the other hand, the numbers of genera in the treatment groups dramatically and significantly reduced $24 \mathrm{~h}$ after danofloxacin injection compared to the control group in this study. However, these numbers returned to the pre-treatment level three days later, which may indicate a transient effect of danofloxacin on microbial richness. Furthermore, a significant clustering difference between pre- and post-treatment microbial communities depicted in the NMDS plot (Figure 2) demonstrated the impact of danofloxacin on the structures of microbial communities. In contrast to the present study, Ferguson and colleagues [33] reported only minor changes in community structure and the absence of changes in microbial diversity following enrofloxacin administration in steers. The discrepancy between these two studies could be partly attributed to the difference in the study designs, where we used ten calves per group under a controlled study setup, and they employed six animals per group. However, it is worthwhile to investigate if the pharmacokinetic differences that exist between danofloxacin and enrofloxacin might have contributed to the variations between these two studies [49].

In the present study, we assessed the effect of danofloxacin on the composition of Campylobacter and its association with the other genera. We were particularly interested in this genus because of the public health significance that some species belonging to Campylobacter have and the rising of antibiotic resistance among them [50]. Twenty-six of the thirty study calves were colonized by Campylobacter species that have public health importance (i.e., C. jejuni) when they were first enrolled in this study, as indicated by bacterial culture (data not shown). Following the inoculation of the laboratory strains, the proportion of Campylobacter (i.e., based on the $16 \mathrm{~S}$ study) continued to rise until the day danofloxacin was administered to the calves in the treatment groups. The increase in the abundance was not quick as there was no significant difference between the sampling time points 1 and 2 (Day 2 and Day 6, the laboratory strains were administered on Day 4 after the start of the study), and the absence of a difference between the two sampling points could be due to the delay in Campylobacter colonization arising from fighting for niches with other microbes [51]. After danofloxacin injection to the treatment calves, the relative abundance of Campylobacter sharply reduced in the samples collected after $24 \mathrm{~h}$; however, it rebounded to the pre-treatment baseline after three days (Figure 5), suggesting that resistant strains emerged as a result of the antibiotic exposure. In agreement with this finding, previous studies conducted by our research team reported the emergence of fluoroquinolone resistance in Campylobacter strains in chicken intestine as early as $24 \mathrm{~h}$ after the initiation of oral enrofloxacin treatment. The proportion of this genus remained unaltered in the control group except for a slight rise in the last sampling day, which strongly suggests that the changes that observed in the treatment groups were driven by danofloxacin administration.

Furthermore, in the current study, we identified 21 genera that had a significant correlation $(p<0.05)$ with Campylobacter. Most of them were confirmed to have a correlation with Campylobacter by the Boruta important variable selection algorithm and the random forest models, which are robust methods to identify correlation between variables [52-54]. Consistent with previous studies, most of the genera in Bacteroidetes phylum had a negative 
correlation and genera in Firmicutes and Proteobacteria phyla had a positive correlation with Campylobacter in the present study [55-57]. However, there are some variations with those studies; for instance, we found a negative correlation between Campylobacter and Prevotella in contrast to the report by Dicksved and colleagues [57]. Understanding the association between gut bacterial taxa has public health implications because gut microbial compositions affect colonization resistance to enteric bacterial infections, including Campylobacter $[50,56]$. For instance, 12 bacterial taxa were shown to have a significant impact on the prevalence and enumeration of E. coli O157:H7 in cattle feces [58]. We also found that the genera that had a correlation with Campylobacter were different before and after danofloxacin injection, except for one genus (Table S4). The change in the patterns of association between gut microbiota may have to do with the way different bacterial taxa respond to antibiotics. As indicated in Tables 2 and 3, some of the bacterial taxa significantly increased or decreased in their compositions following danofloxacin injection, while the other remained unaltered.

The mechanisms by which gut microbiota affect the compositions of Campylobacter include direct inhibition, altering the intraluminal milieu, and production of metabolites [50,57,59]. Exploring the relationship between Campylobacter and gut microbial compositions can give an insight into how to mitigate the spreading and transmission of this foodborne pathogen to humans. Deliberate alteration of gut microbial composition could help to control the amount of Campylobacter shed in cattle feces [58]. Our findings call for further investigation of the identified bacterial taxa with a negative correlation with Campylobacter, particularly those genera with high correlation coefficients or high importance in the random forest plots, which could be used as probiotics to curtail the fecal shedding in cattle or other food animals. Several bacteria, mainly Lactobacillus species, have been shown to reduce colonization and shedding of $C$. jejuni in poultry [60-62]. Brooks and colleagues observed that antibiotic-driven depletion of gut microbiota in a mouse model increased C. jejuni colonization, invasion, and severity of gastroenteritis, which may require administration of probiotics following $C$. jejuni infection to ameliorate inflammation and autoimmune disease [63].

In the current study, the metagenomic Hi-C ProxiMeta predicted the presence of ARGs that confer resistance to tetracycline, aminoglycoside, $\beta$-lactams, and erythromycin in the fecal samples. The presence of ARGs of these antibiotics in the samples is not a surprise because they are among the most frequently used antibiotics on beef farms for the control and prevention of diseases and as feed additives [17]. According to the FDA report, these antibiotics accounted for $67 \%$ (tetracyclines), $12 \%$ ( $\beta$-lactam penicillin), $8 \%$ (macrolides including erythromycin), and 5\% (aminoglycoside) of the total medically important antibiotics approved for use in food-producing animals and actively marketed in 2019 [64]. Furthermore, based on a recently conducted study on 22 U.S. beef farms, macrolide and tetracycline in-feed use accounted for $59.7 \%$ and $19.4 \%$ of the total regimens used in 2016 and 2017, respectively [17]. The in-feed ingredient use of these antibiotics in the feedlot production system contributes toward the emergence and spread of resistance; even, in those farms that stopped using these drugs a long time ago, detection of their resistance genes is a common phenomenon [65]. In agreement with our findings, Zaheer and colleagues [43] documented a high prevalence of tetracycline resistance $(82 \%)$, followed by macrolide (14\%) and aminoglycoside resistance $(2.2 \%)$ in fecal samples from beef cattle.

Fluoroquinolone (in this case danofloxacin) resistance is primarily conferred through a single mutation in gyrA gene that is involved in DNA supercoiling [66]. The method we employed in the current study does not detect a single nucleotide change, despite the rebound of Campylobacter four days post-treatment, suggesting the emergence of resistant strains, which also complemented by previous trials in our laboratory and in the literature $[18,25]$. Genes that have been shown to be associated with fluoroquinolone resistance in other bacteria (e.g., $q n r \mathrm{~A}, q n r \mathrm{~B}, q n r \mathrm{~S}$, etc.) were not detected in the current study. In a previous study that assessed the effect of danofloxacin treatment (IM, for three days) in pigs on the Campylobacter coli resistance profile, the MIC of the isolates increased after the treatment and attributed to the mutation of the gyrA gene [18]. 
In this study, antibiotic resistance determinants have increased both in number and types after danofloxacin administration. The detection of new types of ARGs (i.e., ermF, sat, tet $\mathrm{L}$, and tet $\mathrm{X}$; Table 2) that were not detected in the pre-treatment samples might trigger a question of why resistance genes unrelated to danofloxacin had emerged after the treatment. We propose two hypotheses supported by literature. (1) An enrichment of bacterial strains resistant to these antibiotics: danofloxacin might have wiped out or weakened susceptible bacterial strains and selected for resistance strains to predominate others. The identified resistance genes might have been present before the treatment at a level below the detection limit; however, the treatment might have enriched the bacterial strains hosting these ARGs to the level where our method was capable of detecting them. Parallel to our observation, the sulfonamide resistance gene (sulf 2 ) was enriched following the administration of unassociated antibiotics such as macrolides and tetracycline in cattle [67]. (2) These ARGs might be available at an undetected level before the treatment; however, danofloxacin administration might have favored horizontal gene transfer among genetically related and, probably, unrelated bacteria. Horizontal gene transfer is a common way of acquiring ARGs by bacteria in complex environments, such as in the gut $[27,68,69]$. Co-transfer of ermB and tet $\mathrm{M}$ in Streptococcus pyogenes was reported, which suggested their linkage in individual genetic elements [70]. Colocalization of many ARGs on microbial genomes was found in swine feces and manure; they confer multi-drug resistance to bacteria within a community [71]. In the current study, the exposure to danofloxacin might have enhanced both the dominance of resistant bacteria as well as the transfer of the resistance genes among the microbes in the bacterial communities horizontally [72]. In one study, it was shown that danofloxacin treatment decreased the susceptibility of C. jejuni to macrolide (tylosin) [18]. Meanwhile, in the mouse intestinal microbiota, tetracycline treatment enhanced the abundance of antibiotic ARGs (from 307.3 to 1492.7 ppm), plasmids (from 425.4 to $3235.1 \mathrm{ppm}$ ), and integrons (from 0.8 to $179.6 \mathrm{ppm}$ ) [24].

The ProxiMeta Hi-C metagenomic deconvolution also enabled us to predict bacterial taxa that hosted the ARGs. Previously, using the metagenome-assembled genomes reconstructed by this method, Stalder and colleagues detected an association between ARGs, mobile genetic elements, and host genomes from wastewater samples [36]. In our study, the order Clostridiales in class Clostridia hosted the largest number of ARGs, where ant6, ant9, aph2, sat, tet $\mathrm{O}$, tet $\mathrm{W}$, tet32, tet $\mathrm{W} 40$, and tet 44 were hosted by this bacterium. Sharpea azabuensis DSM_18934 (in the phylum Firmicutes, class Erysipelotrichia, and family Coprobacillaceae) identified in the pre-treatment samples was found to host ant6, ant9, cfr , and tetW. The genus Sharpea was detected in 78 of 80 pre-treatment and 59 of 60 posttreatment samples using the $16 \mathrm{~S}$ rRNA analysis, but it was not identified by the Hi-C metagenomics in the post-treatment samples. Sharpea azabuensis is a ruminal bacterium that produces trans-11 intermediates from linoleic and linolenic acid [73]. Information about the antimicrobial resistance status or acting as a reservoir of resistant genes is not available for this species. In general, the Hi-C findings are consistent with a longitudinal study conducted in China, where they quantified resistance genes in manure from pigs using real-time PCR (i.e., qPCR) and 16S gene sequencing [68]. The authors reported a strong positive correlation between the dynamics of tetracycline resistance genes (i.e., tet $\mathrm{M}$, tet $\mathrm{O}$, tet $\mathrm{Q}$, and tet $\mathrm{W})$ and the relative abundances of certain OTUs of gut associated Clostridiales. Furthermore, higher levels of tet $\mathrm{W}$ and tet 40 were detected in Bacteroidetes following danofloxacin treatment, suggesting the occurrence of ARG transfer horizontally between these Clostridiales in this phylum and other bacteria. Previous studies show that bacterial species in this family acquire resistance genes from other bacteria through transposons and plasmids [74,75]. It appears that danofloxacin administration favors expansion of the ARG host ranges. In this study, 11 and 6 ARG types increased and decreased in copy numbers following the treatment, while 12 and 7 of them had their host range increased and decreased, respectively.

Selected ARGs identified by the metagenomic Hi-C method were further quantified using qPCR in the current study. The results of the latter method were consistent with the 
Hi-C results in that the quantity of tet $\mathrm{W}$ and erm $\mathrm{B}$ were altered significantly, while that of $t e t \mathrm{O}$, tet $\mathrm{X}$, and erm $\mathrm{F}$ were not affected in the post-treatment fecal samples in group $\mathrm{B}$. However, the dramatic increase in tet $\mathrm{W}$ predicted by the $\mathrm{Hi}-\mathrm{C}$ method was not measured by qPCR, which might be attributed to one or both of the following reasons: the fecal samples used in the case of Hi-C ProxiMeta was a pooled sample from two calves on the danofloxacin injection day (i.e., Day 18) for pre-treatment sample and four days later (i.e., Day 22) for the post-treatment sample. In the case of qPCR testing, however, DNA extracts from all calves (ten) in group B were pooled together with a different post-treatment day. The pre-treatment sampling day was the same (i.e., Day 18) but the post-treatment sampling day was different (i.e., Day 28). Nevertheless, the prediction of the trends of ARG changes between the well-established gene quantification method (qPCR) and the novel metagenomic Hi-C method is similar, suggesting the robustness of this novel method in predicting the dynamics of antibiotic determinants in complex microbial communities, such as gut or fecal microbiota.

\section{Materials and Methods}

\subsection{Study Design and Animals}

For this study, 30 dairy calves ( 26 male; 4 female) with predominantly Holstein genetics and no prior history of antibiotic exposure were acquired from a dairy farm located in the state of Iowa. Calves were approximately 8 weeks old at the time of procurement and weighed between $54 \mathrm{~kg}$ to $93 \mathrm{~kg}$. Following purchase, they were group-housed in three rooms (ten calves in each room) at the Livestock Infectious Disease Isolation Facility (LIDIF) at Iowa State University (Biosafety level 2) for 28 days. The rooms were maintained at $20-21^{\circ} \mathrm{C}\left(68-70{ }^{\circ} \mathrm{F}\right)$, and each of them had an independent airflow system. Upon arrival at the facility, calves were visually examined by veterinarians for signs of disease such as lameness, nasal discharge, dyspnea, obtundation, ophthalmic abnormalities, bloat, or diarrhea. Only calves that appeared healthy were enrolled in the study. Following visual examination, calves were weighed and then randomly assigned to one of three study groups after blocking by their weight. Study groups included: Group A-control, oral inoculation with C. jejuni; Group B-oral inoculation with C. jejuni and subcutaneous administration of a single dose of danofloxacin; and Group $\mathrm{C}$-oral inoculation with $C$. jejuni, transtracheal inoculation with Mannheimia haemolytica, and subcutaneous administration of single dose of danofloxacin. Throughout the study course, calves were fed mixed grass hay and a pre-mixed calf starter (Heartland Co-op, Des Moines, IA, USA) and provided with ad libitum water. None of these calves showed serious health problems that required additional antibiotic administration during the study period. The procedures in this study were undertaken following prior approved Institutional Animal Care and Use Committee requirements for Iowa State University.

Following a four-day acclimatization period, all calves were orally inoculated with $C$. jejuni (a cocktail of laboratory strains obtained from Texas, Missouri, and Colorado). The inoculum was prepared by combining fresh colonies of $C$. jejuni with Mueller-Hinton (MH) broth. For each calf, $60 \mathrm{~mL}$ of $C$. jejuni suspension $\left(\sim 10 \times 10^{9} \mathrm{CFU} / \mathrm{mL}\right)$ was administered using a calf esophageal tube. Six days following the administration, calves in group $\mathrm{C}$ were inoculated with $M$. haemolytica ( $10 \mathrm{~mL}$ per calf, $\sim 3 \times 10^{9} \mathrm{CFU} / \mathrm{mL}$ ) by trans-trachea injection using a catheter to induce BRD according to the protocol described in our publication [76]. In the subsequent days, the calves were monitored for signs of BRD, such as elevated body temperature, eye and nasal discharges, ear droop or head tilting, cough, and changes in breathing, eating, and ambulation. Eight days after trans-tracheal inoculation of $M$. haemolytica, calves in groups B and $C$ were subcutaneously injected with a single dose of danofloxacin (ADVOCIN"M, danofloxacin mesylate, Pfizer Animal Health; $8 \mathrm{mg} / \mathrm{kg}$ body weight) in the neck. Fecal samples were collected to a $50 \mathrm{~mL}$ screw-cap tube directly from the rectum from all study calves on days 2, 6, 14, 18, 19, 22, and 28; four times before the treatment (i.e., danofloxacin injection) and three times after the treatment. The calves 
were euthanized with a penetrating captive bolt gun at the end of the study, as per AVMA Guidelines on Euthanasia [77].

\subsection{DNA Extraction, Library Preparation, and Sequencing}

16S rRNA: To determine the effects of danofloxacin on gut microbiota, 16S rRNA analysis was conducted. DNA extractions were performed following ZymoBIOMICS ${ }^{\mathrm{TM}}$ protocol from 210 fecal samples (10 calves per group and seven sampling time points). The V4-V5 hypervariable regions of the bacterial $16 \mathrm{~S}$ rRNA gene were amplified using a universal 16S forward primer (515F: GTGYCAGCMGCCGCGGTAA) and a reverse primer (926R: CCGYCAATTYMTTTRAGTTT). Briefly, the fecal samples were thawed at room temperature for approximately $30 \mathrm{~min}$. From each sample, $200 \mathrm{mg}$ of feces was transferred to a $2 \mathrm{~mL}$ ZR BashingBead ${ }^{\mathrm{TM}}$ lysis tube and mixed with $250 \mu \mathrm{L}$ deionized sterile water, $750 \mu \mathrm{L}$ lysis solution, and $50 \mu \mathrm{L}$ proteinase $\mathrm{K}$. The samples were processed by a bead beater for $10 \mathrm{~min}$ followed by incubation for at least $30 \mathrm{~min}$ in a water bath at $55^{\circ} \mathrm{C}$. Then, the lysis tubes were centrifuged in a microcentrifuge at $10,000 \times g$ for $3 \mathrm{~min}$. The supernatant was harvested to columns and then washed with DNA Wash Buffer 1 and 2. The final product was eluted with DNase/RNase free water, and the concentration of eluted DNA was measured first by NanoDrop 3300 Fluorospectrophotometer (Nanodrop technologies, USA) and confirmed by Qubit fluorometer (Invitrogen). Following normalization of all the DNA extracts, they were transferred to 96-wells plates, and submitted for sequencing to the DNA Facility of Iowa State University. The Earth Microbiome Project protocol was followed for sequencing on the Illumina MiSeq platform $(2 \times 250$ paired-ends $)$ in a single flow cell lane. For control, two community standards were used.

Shotgun sequencing: To assess the effects of danofloxacin on gut microbial resistome, shotgun and ProxiMeta Hi-C metagenomics were performed. For shotgun library preparation, four samples (pooled two pre-treatment samples and pooled two post-treatment samples from calves in Group B) were selected based on the results of 16S analysis. Samples collected right before danofloxacin injection (i.e., Day 18) and four days later (i.e., Day 22) were used for this purpose. Like in the case of 16S rRNA gene, whole-genome DNA was extracted from the fecal samples according to ZymoBIOMICS ${ }^{\mathrm{TM}}$ instructions. The whole-genome extracts were submitted to the DNA Facility of Iowa State University, where a single flow cell lane Illumina HiSeq platform $(2 \times 150 \mathrm{bp})$ was used for sequencing.

Metagenomic Hi-C ProxiMeta DNA extraction and library preparation: The same samples used for the whole-genome shotgun DNA extraction were used for the Metagenomic ProxiMeta sequencing. The Hi-C library was created using a Phase Genomics (Seattle, WA, USA) ProxiMeta Hi-C Microbiome Kit, which is a commercially available version of the Hi-C protocol. Briefly, $100 \mathrm{mg}$ of the fecal sample was washed with TBS, and then genetic material (both chromosomal and non-chromosomal) were crosslinked in vivo while the bacterial cells were still intact using a formaldehyde solution, simultaneously digested using restriction enzymes Sau3AI and MlucI. The genetic materials were proximity ligated with biotinylated nucleotides to create chimeric molecules composed of fragments from different regions of genomes that were physically proximal in vivo according to the manufacturer's instructions for the kit. The chance of inter-cellular interactions of genetic materials was negligible. Molecules were pulled down with streptavidin beads and processed into an Illumina-compatible sequencing library according to the protocol. Sequencing was performed on an Illumina HiSeq instrument $(2 \times 150 \mathrm{bp})$. The bioinformatic analyses were described in our recent publication [78].

Quantitative real-time PCR (qPCR): To assess the dynamics of antimicrobial resistance genes following danofloxacin injection in the fecal samples, we ran qPCR using primers previously designed by Looft et al. [79] presented in Table 5. The target resistance genes were selected based on the metagenomic $\mathrm{Hi}-\mathrm{C}$ results; accordingly, primers were ordered from the ISU DNA facility for tet $\mathrm{W}$, tet $\mathrm{O}$, tet $\mathrm{X}$, erm $\mathrm{B}$, and erm $\mathrm{F}$. The DNA extracts (described above) from fecal samples collected on Day 18 for the pre-treatment and Day 28 for the 
post-treatment, were pooled together for each group separately. PCR assays were run using the SsoAdvanced ${ }^{\mathrm{TM}}$ Universal SYBR ${ }^{\circledR}$ Green Supermix (Bio-Rad, Hercules, CA, USA) and the CFX Maestro ${ }^{\mathrm{TM}}$ Real-Time PCR detection system (Bio-Rad). Dilutions of DNA template for both standards (16S and target genes) and all unknowns were run in triplicate with reaction volumes of $10 \mu \mathrm{L}$. Amplification of DNA occurred with 35 cycles of denaturation at $95^{\circ} \mathrm{C}$ for $10 \mathrm{~s}$ and then annealing for each primer pair at $60^{\circ} \mathrm{C}$ for $30 \mathrm{~s}$. Both standard curves (16S and target genes) were experimentally validated to have high efficiency $>$ $90 \%$ of amplification and precision $\mathrm{R}^{2} \sim 0.98$ prior the analysis. Relative expression was normalized using $16 \mathrm{~S}$ detection levels. The relative fold change of detection between the control and treatment groups (B and C) were calculated using the ISU Gallup Method Equation [80]. Statistical analysis was performed using Dunn pair-wise comparison test in Rstudio to determine significance changes in ARG levels between the pre-and posttreatment pooled samples. An adjusted $p$-value of $<0.05$ was considered significant.

Table 5. Primers used in quantitative real-time PCR tests to assess the dynamics of selected antimicrobial resistance genes in pooled fecal samples from calves.

\begin{tabular}{lllc}
\hline ARGs & Forward Primer & Reverse Primer & Reference \\
\hline erm $\mathrm{B}$ & TGAAAGCCATGCGTCTGACA & CCCTAGTGTTCGGTGAATATCCA & \\
erm $\mathrm{F}$ & TTTCAAAGTGGTGTCAAATATTCCTT & GGACAATGGAACCTCCCAGAA & \\
tetO & ATGTGGATACTACAACGCATGAGATT & TGCCTCCACATGATATTTTCCT & {$[79]$} \\
$t e t \mathrm{~W}$ & TCCTTCCAGTGGCACAGATGT & GCCCCATCTAAAACAGCCAAA & \\
tet $\mathrm{X}$ & AAATTTGTTACCGACACGGAAGTT & CATAGCTGAAAAAATCCAGGACAGTT & \\
\hline
\end{tabular}

\subsection{Bioinformatics and Data Analysis of 165 Data}

Bioinformatic analyses of the $16 \mathrm{~S}$ rRNA sequence data were performed using the QIIME 1.9 and QIIME 2 pipeline. Alpha and beta diversity metrics were computed to evaluate the effects of the antibiotic on the richness and diversity of gut microbiota. The richness was estimated by using observed OTUs, and the evenness using the Shannon index. A non-metric multidimensional scaling plot (distance $=$ bray, $\mathrm{k}=3$ ) was developed using the Vegan $R$ package to visualize the clustering difference between pre- and post-treatment microbial communities.

We used R statistical software for statistical analyses and plotting of graphs. The Kruskal-Wallis test was used to compare the relative abundances of bacterial taxa among the three calf groups and Dunn test for pair-wise comparisons. Similarly, pre- and posttreatment bacterial OTUs were compared by the Wilcoxon signed-rank test. The association of the relative abundance of genus Campylobacter with other genera was explored using the Spearman rank correlation test, and multiple testing correction with a false discovery rate was performed [5]. A $p$-value $<0.05$ was considered significant for all analyses, and for the false discovery rate, $p$ adjusted $<0.05$ was used unless specified otherwise.

\section{Prediction Models Using Random Forest Models}

In total, we obtained 298 genera in our samples; 70 of them with very low relative abundance (zero-inflated) were excluded from further analyses. Among 228 genera, important genera that might have a significant association with pre- and post-treatment status and the compositions of Campylobacter were selected using the Boruta algorithm. For the building of random forest models, the data were randomly divided into a training sub-data $(70 \%)$ and a test sub-data (30\%). The models were first trained using the training sub-data, and then its fitness was evaluated using the test sub-data. The genera selected by the Boruta algorithm were fed to the random forest models to rank the genera based their predictive importance and presented in graphs. The Boruta algorithm and random forest models have been used in the microbiota studies to select and rank important variables from high-dimensional data [52-54]. In this study, we used these methods to identify bacterial taxa that are capable of classifying the microbial communities into pre- and post-treatment, as well as predicting the relative abundance of Campylobacter. For the latter case, further 
analyses were performed by subsetting data as pre-treatment, post-treatment, and control to check if the treatment might have affected the association patterns between genera.

\section{Conclusions}

In summary, our data indicate that subcutaneous administration of danofloxacin significantly altered the compositions and structures of the fecal microbiota in calves. However, not all bacterial taxa were affected alike by the treatment. Several of them showed a reduction in relative abundances, a few other taxa were enriched, and many of them remained the same. The relative abundance of the genus with public health importance, Campylobacter, abruptly reduced $24 \mathrm{~h}$ after the treatment but was resilient after three days, which may imply that this bacterium developed resistance to danofloxacin and, thus, it entails a risk to the public health. The novel ProxiMeta Hi-C method revealed that several types of ARGs that confer resistance to commonly used antibiotics in cattle were enriched by danofloxacin. Additionally, using this method, the presumptive reservoirs of resistance genes have been predicted, and this will help to make further investigations to acquire a deeper understanding of the way resistance genes spread within microbial communities in general and the way they transmit to bacterial species that have public health significance in particular.

Supplementary Materials: The following are available online at https:/ / www.mdpi.com/article/ 10.3390 / microorganisms 9102023 /s1, Table S1: the relative abundance of bacterial phyla of 30 study calves at seven sampling days over four weeks of the study period, Table S2: comparisons of the relative abundance of bacterial taxa between control (group A) and treatments (group B-non-BRDinduced and group C-BRD-induced) groups, Table S3: the Spearman rank correlation test between Campylobacter and other genera in the whole fecal samples (all groups combined), Table S4: the Spearman rank correlation test between Campylobacter and other genera in pre- and post-treatment samples (groups B and C combined), Table S5: summary of antimicrobial resistance genes detected in pre-and post-treatment fecal samples along with their respective bacterial reservoirs in calf group B.

Author Contributions: Conceptualization, Q.Z., P.J.P., O.S. and A.F.B.; methodology, P.J.P., Q.Z., O.S. and A.F.B.; Data collection: A.F.B., D.B.-G., T.H., C.S., R.D. and G.D.; Laboratory work, A.F.B., T.H., C.S., B.R., A.H. and D.B.-G.; formal analysis, P.J.P. and A.F.B.; writing-original draft preparation, A.F.B. and P.J.P.; writing-review and editing, all; supervision, G.D., R.D., P.J.P., O.S. and A.F.B.; project administration, Q.Z. and P.J.P.; funding acquisition, Q.Z., P.J.P., O.S. and G.D. All authors have read and agreed to the published version of the manuscript.

Funding: This work was supported by the Agriculture and Food Research Initiative's Competitive Grant 2017-68003-26499 from the USDA National Institute of Food and Agriculture.

Institutional Review Board Statement: The procedures in this study were undertaken following prior approved Institutional Animal Care and Use Committee requirements for Iowa State University.

Informed Consent Statement: Not applicable.

Data Availability Statement: Data are available upon request.

Acknowledgments: Ivan Liachko and Max Press from Phase Genomics provided us support in ProxiMeta Hi-C metagenomics.

Conflicts of Interest: The authors declare no conflict of interest.

\section{References}

1. Wichmann, F.; Udikovic-Kolic, N.; Andrew, S.; Handelsman, J. Diverse Antibiotic Resistance Genes in Dairy Cow Manure. Mbio 2014, 5, e01017-13. [CrossRef]

2. Zhu, Y.G.; Johnson, T.A.; Su, J.Q.; Qiao, M.; Guo, G.X.; Stedtfeld, R.D.; Hashsham, S.A.; Tiedje, J.M. Diverse and abundant antibiotic resistance genes in Chinese swine farms. Proc. Natl. Acad. Sci. USA 2013, 110, 3435-3440. [CrossRef]

3. Call, D.R.; Davis, M.A.; Sawant, A.A. Antimicrobial resistance in beef and dairy cattle production. Anim. Health Res. Rev. 2008, 9 , 159-167. [CrossRef] 
4. Catry, B.; Dewulf, J.; Maes, D.; Pardon, B.; Callens, B.; Vanrobaeys, M.; Opsomer, G.; de Kruif, A.; Haesebrouck, F. Effect of Antimicrobial Consumption and Production Type on Antibacterial Resistance in the Bovine Respiratory and Digestive Tract. PLoS ONE 2016, 11, e0146488. [CrossRef] [PubMed]

5. Ocejo, M.; Oporto, B.; Hurtado, A. 16S rRNA amplicon sequencing characterization of caecal microbiome composition of broilers and free-range slow-growing chickens throughout their productive lifespan. Sci. Rep. 2019, 9, 2506. [CrossRef]

6. O'Hara, A.M.; Shanahan, F. The gut flora as a forgotten organ. Embo Rep. 2006, 7, 688-693. [CrossRef]

7. Udikovic-Kolic, N.; Wichmann, F.; Broderick, N.A.; Handelsman, J. Bloom of resident antibiotic-resistant bacteria in soil following manure fertilization. Proc. Natl. Acad. Sci. USA 2014, 111, 15202-15207. [CrossRef] [PubMed]

8. Bennani, H.; Mateus, A.; Mays, N.; Eastmure, E.; Staerk, K.D.C.; Hasler, B. Overview of Evidence of Antimicrobial Use and Antimicrobial Resistance in the Food Chain. Antibiotics 2020, 9, 49. [CrossRef] [PubMed]

9. Johnson, K.K.; Pendell, D.L. Market Impacts of Reducing the Prevalence of Bovine Respiratory Disease in United States Beef Cattle Feedlots. Front. Vet. Sci. 2017, 4, 189. [CrossRef] [PubMed]

10. Smith, A.B.; Renter, D.G.; Cernicchiaro, N.; Shi, X.R.; Nickell, J.S.; Keil, D.J.; Nagaraja, T.G. A Randomized Trial to Assess the Effect of Fluoroquinolone Metaphylaxis on the Fecal Prevalence and Quinolone Susceptibilities of Salmonella and Campylobacter in Feedlot Cattle. Foodborne Pathog. Dis. 2017, 14, 600-607. [CrossRef] [PubMed]

11. Hilton, W.M. BRD in 2014: Where have we been, where are we now, and where do we want to go? Anim. Health Res. Rev. 2014, 15, 120-122. [CrossRef] [PubMed]

12. USDA. Feedlot 2011 Part I: Management Practices on U.S. Feedlots with a Capacity of 1,000 or More Head; USDA-APHIS-VS-CEAHNAHMS, Fort Collins, CO.; USDA: Washington, DC, USA, 2013.

13. Guterbock, W.M. The impact of BRD: The current dairy experience. Anim. Health Res. Rev. 2014, 15, 130-134. [CrossRef]

14. USDA. Dairy 2007: Part V: Changes in Dairy Cattle Health and Management Practices in the United States, 1996-2007; USDA-APHIS National Animal Health Monitoring System. Info Sheet; USDA: Washington, DC, USA, 2009.

15. Taylor, J.D.; Fulton, R.W.; Lehenbauer, T.W.; Step, D.L.; Confer, A.W. The epidemiology of bovine respiratory disease: What is the evidence for predisposing factors? Can. Vet. J. -Rev. Vet. Can. 2010, 51, 1095-1102.

16. FDA. 2018 Summary Report on Antimicrobials Sold or Distributed for Use in Food-Producing Animals; FDA: Rockville, MD, USA, 2019.

17. Hope, K.J.; Apley, M.D.; Schrag, N.F.D.; Lubbers, B.V.; Singer, R.S. Antimicrobial use in 22 US beef feedyards: 2016-2017. Zoonoses Public Health 2020, 67, 94-110. [CrossRef]

18. Juntunen, P.; Olkkola, S.; Hanninen, M.-L. Longitudinal on-farm study of the development of antimicrobial resistance in Campylobacter coli from pigs before and after danofloxacin and tylosin treatments. Vet. Microbiol. 2011, 150, 322-330. [CrossRef]

19. Dryden, M.S.; Gabb, R.J.E.; Wright, S.K. Empirical Treatment of Severe Acute Community-Acquired Gastroenteritis with Ciproftoxacin. Clin. Infect. Dis. 1996, 22, 1019-1025. [CrossRef]

20. Nelson, J.M.; Chiller, T.M.; Powers, J.H.; Angulo, F.J. Fluoroquinolone-resistant Campylobacter species and the withdrawal of fluoroquinolones from use in poultry: A public health success story. Clin. Infect. Dis. 2007, 44, 977-980. [CrossRef]

21. Foditsch, C.; Pereira, R.V.V.; Siler, J.D.; Altier, C.; Warnick, L.D. Effects of treatment with enrofloxacin or tulathromycin on fecal microbiota composition and genetic function of dairy calves. PLoS ONE 2019, 14, e219635. [CrossRef]

22. Wieczorek, K.; Osek, J. Antimicrobial resistance mechanisms among Campylobacter. BioMed Res. Int. 2013, $2013,340605$. [CrossRef] [PubMed]

23. Kyselkova, M.; Kotrbova, L.; Bhumibhamon, G.; Chronakova, A.; Jirout, J.; Vrchotovaa, N.; Schmitt, H.; Elhottovaa, D. Tetracycline resistance genes persist in soil amended with cattle feces independently from chlortetracycline selection pressure. Soil Biol. Biochem. 2015, 81, 259-265. [CrossRef]

24. Yin, J.B.; Zhang, X.X.; Wu, B.; Xian, Q.M. Metagenomic insights into tetracycline effects on microbial community and antibiotic resistance of mouse gut. Ecotoxicology 2015, 24, 2125-2132. [CrossRef]

25. Cheng, P.; Yang, Y.; Li, F.; Li, X.; Liu, H.; Fazilani, S.A.; Guo, W.; Xu, G.; Zhang, X. The prevalence and mechanism of fluoroquinolone resistance in Escherichia coli isolated from swine farms in China. BMC Vet. Res. 2020, 16, 258. [CrossRef]

26. Li, J.; Hao, H.; Cheng, G.; Liu, C.; Ahmed, S.; Shabbir, M.A.B.; Hussain, H.I.; Dai, M.; Yuan, Z. Microbial Shifts in the Intestinal Microbiota of Salmonella Infected Chickens in Response to Enrofloxacin. Front. Microbiol. 2017, 8, 1711. [CrossRef]

27. Francino, M.P. Antibiotics and the human gut microbiome: Dysbioses and accumulation of resistances. Front. Microbiol. 2016, 7 , 01543. [CrossRef]

28. Panda, S.; El Khader, I.; Casellas, F.; Vivancos, J.L.; Cors, M.G.; Santiago, A.; Cuenca, S.; Guarner, F.; Manichanh, C. Short-Term Effect of Antibiotics on Human Gut Microbiota. PLoS ONE 2014, 9, e95476. [CrossRef]

29. Schokker, D.; Jansman, A.J.M.; Veninga, G.; de Bruin, N.; Vastenhouw, S.A.; de Bree, F.M.; Bossers, A.; Rebel, J.M.J.; Smits, M.A. Perturbation of microbiota in one-day old broiler chickens with antibiotic for 24 hours negatively affects intestinal immune development. BMC Genom. 2017, 18, 241. [CrossRef]

30. Schokker, D.; Zhang, J.; Zhang, L.-1.; Vastenhouw, S.A.; Heilig, H.G.H.J.; Smidt, H.; Rebel, J.M.J.; Smits, M.A. Early-Life Environmental Variation Affects Intestinal Microbiota and Immune Development in New-Born Piglets. PLoS ONE 2014, 9, e100040. [CrossRef]

31. Zaura, E.; Brandt, B.W.; de Mattos, M.J.T.; Buijs, M.J.; Caspers, M.P.M.; Rashid, M.-U.; Weintraub, A.; Nord, C.E.; Savell, A.; Hu, Y.; et al. Same Exposure but Two Radically Different Responses to Antibiotics: Resilience of the Salivary Microbiome versus Long-Term Microbial Shifts in Feces. Mbio 2015, 6, e01693-15. [CrossRef] [PubMed] 
32. Gronvold, A.M.R.; Mao, Y.J.; L'Abee-Lund, T.M.; Sorum, H.; Sivertsen, T.; Yannarell, A.C.; Mackie, R.I. Fecal microbiota of calves in the clinical setting: Effect of penicillin treatment. Vet. Microbiol. 2011, 153, 354-360. [CrossRef]

33. Ferguson, K.M.; Jacob, M.E.; Theriot, C.M.; Callahan, B.J.; Prange, T.; Papich, M.G.; Foster, D.M. Dosing Regimen of Enrofloxacin Impacts Intestinal Pharmacokinetics and the Fecal Microbiota in Steers. Front. Microbiol. 2018, 9, 2190. [CrossRef]

34. Weese, J.S.; Jelinski, M. Assessment of the Fecal Microbiota in Beef Calves. J. Vet. Intern. Med. 2017, 31, 176-185. [CrossRef]

35. Durso, L.M.; Harhay, G.P.; Smith, T.P.L.; Bono, J.L.; DeSantis, T.Z.; Harhay, D.M.; Andersen, G.L.; Keen, J.E.; Laegreid, W.W.; Clawson, M.L. Animal-to-Animal Variation in Fecal Microbial Diversity among Beef Cattle. Appl. Environ. Microbiol. 2010, 76, 4858-4862. [CrossRef]

36. Stalder, T.; Press, M.O.; Sullivan, S.; Liachko, I.; Top, E.M. Linking the resistome and plasmidome to the microbiome. ISME J. 2019, 13, 2437-2446. [CrossRef] [PubMed]

37. Mzyk, D.A.; Bublitz, C.M.; Martinez, M.N.; Davis, J.L.; Baynes, R.E.; Smith, G.W. Impact of bovine respiratory disease on the pharmacokinetics of danofloxacin and tulathromycin in different ages of calves. PLoS ONE 2019, 14, e0218864. [CrossRef] [PubMed]

38. Mestorino, N.; Marchetti, M.L.; Turic, E.; Pesoa, J.; Errecalde, J. Concentrations of danofloxacin 18\% solution in plasma, milk and tissues after subcutaneous injection in dairy cows. Anal. Chim. Acta 2009, 637, 33-39. [CrossRef] [PubMed]

39. Ferran, A.A.; Bibbal, D.; Pellet, T.; Laurentie, M.; Gicquel-Bruneau, M.; Sanders, P.; Schneider, M.; Toutain, P.L.; Bousquet-Melou, A. Pharmacokinetic/pharmacodynamic assessment of the effects of parenteral administration of a fluoroquinolone on the intestinal microbiota: Comparison of bactericidal activity at the gut versus the systemic level in a pig model. Int. J. Antimicrob. Agents 2013, 42, 429-435. [CrossRef] [PubMed]

40. Khoder, M.; Tsapis, N.; Domergue-Dupont, V.; Gueutin, C.; Fattal, E. Removal of residual colonic ciprofloxacin in the rat by activated charcoal entrapped within zinc-pectinate beads. Eur. J. Pharm. Sci. 2010, 41, 281-288. [CrossRef] [PubMed]

41. Edlund, C.; Lindqvist, L.; Nord, C.E. Binding of norfloxacin to human fecal material. Rev. Infect. Dis. 1989, 11, S1237-S1238.

42. Wiuff, C.; Lykkesfeldt, J.; Svendsen, O.; Aarestrup, F.M. The effects of oral and intramuscular administration and dose escalation of enrofloxacin on the selection of quinolone resistance among Salmonella and coliforms in pigs. Res. Vet. Sci. 2003, 75, 185-193. [CrossRef]

43. Zaheer, R.; Lakin, S.M.; Polo, R.O.; Cook, S.R.; Larney, F.J.; Morley, P.S.; Booker, C.W.; Hannon, S.J.; Van Domselaar, G.; Read, R.R.; et al. Comparative diversity of microbiomes and Resistomes in beef feedlots, downstream environments and urban sewage influent. BMC Microbiol. 2019, 19, 197. [CrossRef]

44. Durso, L.; Wells, J.; Kim, M.S. Diversity of Microbiomes in Beef Cattle. In Encyclopedia of Metagenomics; Nelson, K., Ed.; Springer: New York, NY, USA, 2014.

45. Valerio de Oliveira, M.N.; Jewell, K.A.; Freitas, F.S.; Benjamin, L.A.; Totola, M.R.; Borges, A.C.; Moraes, C.A.; Suen, G. Characterizing the microbiota across the gastrointestinal tract of a Brazilian Nelore steer. Vet. Microbiol. 2013, 164, 307-314. [CrossRef]

46. Kim, M.; Kim, J.; Kuehn, L.A.; Bono, J.L.; Berry, E.D.; Kalchayanand, N.; Freetly, H.C.; Benson, A.K.; Wells, J.E. Investigation of bacterial diversity in the feces of cattle fed different diets. J. Anim. Sci. 2014, 92, 683-694. [CrossRef]

47. Rudi, K.; Moen, B.; Sekelja, M.; Frisli, T.; Lee, M.R.F. An eight-year investigation of bovine livestock fecal microbiota. Vet. Microbiol. 2012, 160, 369-377. [CrossRef]

48. Qiu, Q.; Zhu, Y.; Qiu, X.; Gao, C.; Wang, J.; Wang, H.; He, Y.; Ur Rahman, M.A.; Cao, B.; Su, H. Dynamic Variations in Fecal Bacterial Community and Fermentation Profile of Holstein Steers in Response to Three Stepwise Density Diets. Animals 2019, 9 , 560. [CrossRef]

49. McKellar, Q.; Gibson, I.; Monteiro, A.; Bregante, M. Pharmacokinetics of enrofloxacin and danofloxacin in plasma, inflammatory exudate, and bronchial secretions of calves following subcutaneous administration. Antimicrob. Agents Chemother. 1999, 43, 1988-1992. [CrossRef]

50. Brooks, P.T.; Mansfield, L.S. Effects of antibiotic resistance (AR) and microbiota shifts on Campylobacter jejuni-mediated diseases. Anim. Health Res. Rev. 2017, 18, 99-111. [CrossRef]

51. O'Loughlin, J.L.; Samuelson, D.R.; Braundmeier-Fleming, A.G.; White, B.A.; Haldorson, G.J.; Stone, J.B.; Lessmann, J.J.; Eucker, T.P.; Konkel, M.E. The Intestinal Microbiota Influences Campylobacter jejuni Colonization and Extraintestinal Dissemination in Mice. Appl. Environ. Microbiol. 2015, 81, 4642-4650. [CrossRef]

52. Degenhardt, F.; Seifert, S.; Szymczak, S. Evaluation of variable selection methods for random forests and omics data sets. Brief. Bioinform. 2019, 20, 492-503. [CrossRef]

53. Chen, J.; Chia, N.; Kalari, K.R.; Yao, J.Z.; Novotna, M.; Soldan, M.M.P.; Luckey, D.H.; Marietta, E.V.; Jeraldo, P.R.; Chen, X.F.; et al. Multiple sclerosis patients have a distinct gut microbiota compared to healthy controls. Sci. Rep. 2016, 6, 28484. [CrossRef] [PubMed]

54. Subramanian, S.; Huq, S.; Yatsunenko, T.; Haque, R.; Mahfuz, M.; Alam, M.A.; Benezra, A.; DeStefano, J.; Meier, M.F.; Muegge, B.D.; et al. Persistent gut microbiota immaturity in malnourished Bangladeshi children. Nature 2014, 510, 417-421. [CrossRef]

55. Sakaridis, I.; Ellis, R.J.; Cawthraw, S.A.; van Vliet, A.H.M.; Stekel, D.J.; Penell, J.; Chambers, M.; La Ragione, R.M.; Cook, A.J. Investigating the Association Between the Caecal Microbiomes of Broilers and Campylobacter Burden. Front. Microbiol. 2018, 9 , 927. [CrossRef] [PubMed]

56. Kampmann, C.; Dicksved, J.; Engstrand, L.; Rautelin, H. Composition of human faecal microbiota in resistance to Campylobacter infection. Clin. Microbiol. Infect. 2016, 22, 61.e1-61.e8. [CrossRef] [PubMed] 
57. Dicksved, J.; Ellstrom, P.; Engstrand, L.; Rautelin, H. Susceptibility to Campylobacter Infection Is Associated with the Species Composition of the Human Fecal Microbiota. Mbio 2014, 5, e01212-14. [CrossRef] [PubMed]

58. Kim, M.; Kuehn, L.A.; Bono, J.L.; Berry, E.D.; Kalchayanand, N.; Freetly, H.C.; Benson, A.K.; Wells, J.E. The impact of the bovine faecal microbiome on Escherichia coli O157:H7 prevalence and enumeration in naturally infected cattle. J. Appl. Microbiol. 2017, 123, 1027-1042. [CrossRef]

59. Garber, J.M.; Nothaft, H.; Pluvinage, B.; Stahl, M.; Bian, X.; Porfirio, S.; Enriquez, A.; Butcher, J.; Huang, H.; Glushka, J.; et al. The gastrointestinal pathogen Campylobacter jejuni metabolizes sugars with potential help from commensal Bacteroides vulgatus. Commun. Biol. 2020, 3, 1-11. [CrossRef]

60. Morishita, T.Y.; Aye, P.P.; Harr, B.S.; Cobb, C.W.; Clifford, J.R. Evaluation of an avian-specific probiotic to reduce the colonization and shedding of Campylobacter jejuni in broilers. Avian Dis. 1997, 41, 850-855. [CrossRef]

61. Ghareeb, K.; Awad, W.A.; Mohnl, M.; Porta, R.; Biarnes, M.; Bohm, J.; Schatzmayr, G. Evaluating the efficacy of an avian-specific probiotic to reduce the colonization of Campylobacter jejuni in broiler chickens. Poult. Sci. 2012, 91, 1825-1832. [CrossRef]

62. Santini, C.; Baffoni, L.; Gaggia, F.; Granata, M.; Gasbarri, R.; Di Gioia, D.; Biavati, B. Characterization of probiotic strains: An application as feed additives in poultry against Campylobacter jejuni. Int. J. Food Microbiol. 2010, 141, S98-S108. [CrossRef]

63. Brooks, P.T.; Bell, J.A.; Bejcek, C.E.; Malik, A.; Mansfield, L.S. An antibiotic depleted microbiome drives severe Campylobacter jejuni-mediated Type 1/17 colitis, Type 2 autoimmunity and neurologic sequelae in a mouse model. J. Neuroimmunol. 2019, 337, 577048. [CrossRef]

64. FDA. 2019 Summary Report on Antimicrobials Sold or Distributed for Use in Food-Producing Animals; FDA: Rockville, MD, USA, 2020.

65. Rovira, P.; McAllister, T.; Lakin, S.M.; Cook, S.R.; Doster, E.; Noyes, N.R.; Weinroth, M.D.; Yang, X.; Parker, J.K.; Boucher, C.; et al. Characterization of the Microbial Resistome in Conventional and "Raised Without Antibiotics" Beef and Dairy Production Systems. Front. Microbiol. 2019, 10, 1980. [CrossRef]

66. Jacoby, G.A. Mechanisms of resistance to quinolones. Clin. Infect. Dis. 2005, 41, S120-S126. [CrossRef] [PubMed]

67. Holman, D.B.; Yang, W.; Alexander, T.W. Antibiotic treatment in feedlot cattle: A longitudinal study of the effect of oxytetracycline and tulathromycin on the fecal and nasopharyngeal microbiota. Microbiome 2019, 7, 1-14. [CrossRef]

68. Leclercq, S.O.; Wang, C.; Sui, Z.; Wu, H.; Zhu, B.; Deng, Y.; Feng, J. A multiplayer game: Species of Clostridium, Acinetobacter, and Pseudomonas are responsible for the persistence of antibiotic resistance genes in manure-treated soils. Environ. Microbiol. 2016, 18, 3494-3508. [CrossRef] [PubMed]

69. Slizovskiy, I.B.; Mukherjee, K.; Dean, C.J.; Boucher, C.; Noyes, N.R. Mobilization of Antibiotic Resistance: Are Current Approaches for Colocalizing Resistomes and Mobilomes Useful? Front. Microbiol. 2020, 11, 1376. [CrossRef] [PubMed]

70. Brenciani, A.; Bacciaglia, A.; Vecchi, M.; Vitali, L.A.; Varaldo, P.E.; Giovanettil, E. Genetic elements carrying erm(B) in Streptococcus pyogenes and association with tet(M) tetracycline resistance gene. Antimicrob. Agents Chemother. 2007, 51, 1209-1216. [CrossRef] [PubMed]

71. Johnson, T.A.; Stedtfeld, R.D.; Wang, Q.; Cole, J.R.; Hashsham, S.A.; Looft, T.; Zhu, Y.-G.; Tiedje, J.M. Clusters of Antibiotic Resistance Genes Enriched Together Stay Together in Swine Agriculture. Mbio 2016, 7, e02214-15. [CrossRef] [PubMed]

72. Shin, S.W.; Shin, M.K.; Jung, M.; Belaynehe, K.M.; Yoo, H.S. Prevalence of Antimicrobial Resistance and Transfer of Tetracycline Resistance Genes in Escherichia coli Isolates from Beef Cattle. Appl. Environ. Microbiol. 2015, 81, 5560-5566. [CrossRef]

73. Dewanckele, L.; Vlaeminck, B.; Fievez, V. Sharpea azabuensis: A ruminal bacterium that produces trans- 11 intermediates from linoleic and linolenic acid. Microbiology 2019, 165, 772-778. [CrossRef]

74. Whittle, G.; Shoemaker, N.B.; Salyers, A.A. The role of Bacteroides conjugative transposons in the dissemination of antibiotic resistance genes. Cell. Mol. Life Sci. 2002, 59, 2044-2054. [CrossRef]

75. Quesada-Gomez, C. Bacteroides mobilizable and conjugative genetic elements: Antibiotic resistance among clinical isolates. Rev. Esp. De Quimioter. 2011, 24, 184-190.

76. Hanthorn, C.J.; Dewell, R.D.; Cooper, V.L.; Frana, T.S.; Plummer, P.J.; Wang, C.; Dewell, G.A. Randomized clinical trial to evaluate the pathogenicity of Bibersteinia trehalosi in respiratory disease among calves. BMC Vet. Res. 2014, 10, 1-8. [CrossRef] [PubMed]

77. AVMA. AVMA Guidelines for the Euthanasia of Animals: Version 2020.0.1, 2020 Edition; AVMA: Schaumburg, IL, USA, 2020.

78. Beyi, A.F.; Hassall, A.; Phillips, G.J.; Plummer, P.J. Tracking Reservoirs of Antimicrobial Resistance Genes in a Complex Microbial Community Using Metagenomic Hi-C: The Case of Bovine Digital Dermatitis. Antibiotics 2021, 10, 221. [CrossRef]

79. Looft, T.; Johnson, T.A.; Allen, H.K.; Bayles, D.O.; Alt, D.P.; Stedtfeld, R.D.; Sul, W.J.; Stedtfeld, T.M.; Chai, B.; Cole, J.R.; et al. In-feed antibiotic effects on the swine intestinal microbiome. Proc. Natl. Acad. Sci. USA 2012, 109, 1691. [CrossRef] [PubMed]

80. Gallup, J.M.; Ackermann, M.R. Addressing fluorogenic real-time qPCR inhibition using the novel custom Excel file system 'FocusField2-6GallupqPCRSet-upTool-001' to attain consistently high fidelity qPCR reactions. Biol. Proced. Online 2006, 8, 87-151. [CrossRef] [PubMed] 